\title{
In-cell infection: a novel pathway for Epstein-Barr virus infection mediated by cell-in-cell structures
}

\begin{abstract}
Chao $\mathrm{Ni}^{1, *}$, Yuhui Chen ${ }^{1, *}$, Musheng Zeng ${ }^{2, *}$, Rongjuan $\mathrm{Pei}^{3}$, Yong $\mathrm{Du}^{2}$, Linquan Tang ${ }^{2}$, Mengyi Wang ${ }^{4}$, Yazhuo $\mathrm{Hu}^{1}$, Hanyu Zhu ${ }^{1}$, Meifang $\mathrm{He}^{1}$, Xiawei $\mathrm{Wei}^{5}$, Shan Wang ${ }^{6}$, Xiangkai Ning ${ }^{7}$, Manna Wang ${ }^{7}$, Jufang Wang ${ }^{1}$, Li Ma ${ }^{8}$, Xinwen Chen ${ }^{3}$, Qiang Sun ${ }^{7}$, Hong Tang ${ }^{3}$, Ying Wang ${ }^{9}$, Xiaoning Wang ${ }^{1}$

${ }^{1}$ Institute of Life Sciences, Chinese PLA General Hospital and School of Bioscience and Bioengineering, South China University of Technology, Key Laboratory of Normal aging and Geriatric \& the State Key Laboratory of Kidney, Beijing 100853 \& the Provincial Key Laboratory of Biotechnology, Guangdong 510006, China; ${ }^{2}$ State Key Laboratory of Oncology in Southern China, Sun Yat-Sen University Cancer Center, Guangzhou, Guangdong 510060, China $;{ }^{3}$ Wuhan Institute of Virology, Chinese Academy of Sciences, Wuhan, Hubei 430071, China; ${ }^{4}$ School of Ophthalmology and Optometry, Wenzhou Medical University, Wenzhou, Zhejiang 325000, China; ${ }^{5}$ State Key Laboratory of Biotherapy, West China Hospital, Sichuan University, Chengdu, Sichuan 610041, China; ${ }^{6}$ The Second Affiliated Hospital, Guangzhou Medical University, Guangzhou, Guangdong 510260, China; ${ }^{7}$ Laboratory of Cell Engineering, Institute of Biotechnology, Beijing 100071, China; ${ }^{8}$ Institute of Molecular Immunology, School of Biotechnology, Southern Medical University, Guangzhou, Guangdong 510515, China; ${ }^{9}$ Department of Immunology and Microbiology, Shanghai Institute of Immunology, Shanghai Jiaotong University School of Medicine, Shanghai 200025, China
\end{abstract}

Epstein-Barr virus (EBV) can infect both susceptible B lymphocytes and non-susceptible epithelial cells (ECs). Viral tropism analyses have revealed two intriguing means of EBV infection, either by a receptor-mediated infection of $B$ cells or by a cell-to-cell contact-mediated infection of non-susceptible ECs. Herein, we report a novel "in-cell infection" mechanism for EBV infection of non-susceptible ECs through the formation of cell-in-cell structures. Epithelial CNE-2 cells were invaded by EBV-infected Akata B cells to form cell-in-cell structures in vitro. Such unique cellular structures could be readily observed in the specimens of nasopharyngeal carcinoma. Importantly, the formation of cell-in-cell structures led to the autonomous activation of EBV within Akata cells and subsequent viral transmission to CNE-2 cells, as evidenced by the expression of viral genes and the presence of virion particles in CNE-2 cells. Significantly, EBV generated from in-cell infected ECs displayed altered tropism with higher infection efficacy to both $B$ cells and ECs. In addition to CNE-2 tumor cells, cell-in-cell structure formation could also mediate EBV infection of NPEC1-Bmi1 cells, an immortalized nasopharyngeal epithelial cell line. Furthermore, efficient infection by this mechanism involved the activation of the PI3K/AKT signaling pathway. Thus, our study identified "in-cell infection" as a novel mechanism for $\mathrm{EBV}$ infection. Given the diversity of virus-infected cells and the prevalence of cell-in-cell structures during chronic infection, we speculate that "in-cell infection" is likely a general mechanism for EBV and other viruses to infect non-susceptible ECs.

Keywords: cell-in-cell structures; in-cell infection; Epstein-Barr virus; tropism; PI3K/AKT pathway

Cell Research (2015) 25:785-800. doi:10.1038/cr.2015.50; published online 28 April 2015

*These three authors contributed equally to this work.

Correspondence: Xiaoning Wang ${ }^{\mathrm{a}}$, Ying Wang ${ }^{\mathrm{b}}$

${ }^{a}$ E-mail: xnwang88@163.com

bE-mail: ywang@sibs.ac.cn

Received 12 January 2015; revised 14 February 2015; accepted 10 March

2015; published online 28 April 2015

\section{Introduction}

Viral infections remain a threat to global health and cause either acute fatal diseases such as Ebola [1] and severe acute respiratory syndrome [2] or chronic diseases such as acquired immune deficiency syndrome (AIDS) [3] and hepatitis B [4]. Moreover, certain persistent viral infections have been implicated in tumorigenesis, like cervical carcinoma caused by human papillomavirus [5] 
or nasopharyngeal carcinoma (NPC) and Burkitt's lymphoma by Epstein-Barr virus (EBV) $[6,7]$.

Viral infection displays strict species and cell type specificity, a definition called viral tropism [8], which is crucial in determining the manifestation and outcome of viral infection [9-11]. However, not rare but quite often, viruses gain the ability to infect non-susceptible cells, as shown by human immunodeficiency virus (HIV) infection of the genital and gastrointestinal cells [12, 13], making the clinical prophylaxis and therapy more complex. Therefore, studies on the mechanisms of virus tropism are critical for developing alternative anti-virus strategies $[14,15]$.

EBV can infect both susceptible B lymphocytes and non-susceptible nasopharyngeal epithelial cells (ECs) in vitro and in vivo $[16,17]$. EBV infection of B lymphocytes is a typical receptor-mediated process and represents a mechanism for the establishment of longterm latency in the host $[17,18]$. Such susceptibility is believed to be conferred by an interaction between the viral envelope glycoprotein gp350/220 [19] and the complement receptor $2(\mathrm{CR} 2) / \mathrm{CD} 21$, the primary receptor for EBV on B cells [20, 21]. Receptor-mediated internalization of EBV requires the interaction of another viral envelope glycoprotein gp42 with HLA class II molecules on $\mathrm{B}$ cells, which thereafter initiates the fusion process involving the core fusion complex, gH/gL/gp42 [22, 23]. This receptor-mediated process is cell type specific, making viral receptors on host cells therapeutic targets [2426].

EBV infection of ECs in vivo has been observed for over three decades $[27,28]$, with its underlying mechanisms still being actively investigated. In contrast to $\mathrm{B}$ cells, ECs express the viral receptor CR2/CD21 at very low levels and do not constitutively express HLA class II molecules [29]. Therefore, the infection of ECs in vitro by cell-free EBV virions is not as efficient as that of B cells. Nevertheless, ECs can be efficiently infected through co-culturing with Akata cells (an EBV-producing B lymphoblastoid cell line), which is thought to be mediated by a mechanism termed cell-to-cell infection [30]. This process involves EBV binding to B cells through CR2/CD21, activation of adhesion molecules and the subsequent entry of EBV into ECs [31, 32]. Such an intercellular conjugation requires the interaction between CD11b on EBV-loaded B cells and the heparin moiety of CD44v3 and LEEP-CMA on the basolateral surface of ECs [32]. Interestingly, cell-to-cell infection rates vary among different types of target cells, ranging from $0 \%$ to $25 \%[30,31]$. More recently, it was reported that cell-to-cell transmission was efficient in mediating EBV infection of raft cultures generated from primary human keratinocytes in vitro, resulting in productive viral replication in organotypic cultures and the release of viral particles to intercellular space [33]. Of note, cell-to-cell infection is not unique to EBV. It also accounts for the efficient transmission of HIV between susceptible CD4 ${ }^{+}$ $\mathrm{T}$ cells $[34,35]$, representing a mechanism for resistance to anti-viral therapy and subsequent viral persistence [36, 37].

Cell-to-cell infection explains how EBV infects non-susceptible ECs efficiently. It remains interesting to explore other types of EBV infection of ECs. We recently reported the existence of unique cell-in-cell structures in clinical cancer specimens, in which lymphocytes penetrated both malignant and normal ECs [38]. Cell-in-cell structure was noted over 100 years ago and has recently aroused great interest due to its potential pathophysiological significance $[39,40]$. Cell-in-cell structures can occur either homotypically or heterotypically. Heterotypic cell-in-cell structures, formed by the invasion of lymphocytes into tumor cells of epithelial origin, were found to occur more prevalently than homotypic structures both in vitro and in vivo [38]. The fates of the invading cells and host cells in cell-in-cell structures are diverse [4143]. Most often the invading lymphocytes die by entosis or emperitosis [44]. Entosis is a lysosome-dependent cell death and involves the activation of the Rho/Rock signal pathway [45]. Emperitosis is a caspase 3-dependent apoptotic process [40]. Only immune cells with cytotoxicity undergo emperitosis. Non-cytotoxic cells, like B cells, undergo entosis. Penetration of lymphocytes into ECs in specimens from patients with NPC [46] and in liver sections of mouse hepatitis models [38] has been previously reported. However, it is not clear whether the formation of the cell-in-cell structures between ECs and lymphocytes infected by certain viruses such as EBV or HIV plays certain roles in mediating virus transmission to non-susceptible ECs.

To investigate this possibility, we searched for cell-incell structures formed by lymphocytes and ECs in NPC specimens. The in vitro study using Burkett's lymphoma (BL)-derived EBV-eGFP Akata cells [47] and a typical EBV non-susceptible EC cell line CNE-2 [48] revealed that the formation of heterotypic cell-in-cell structures facilitated EBV transmission from EBV-infected Akata cells to non-infected CNE-2 cells. These new findings suggest that cell-in-cell infection, in addition to cell-tocell infection, plays a role in transmitting viruses from host cells to non-susceptible ECs. Interestingly, EBV viral particles produced via the cell-in-cell process possessed broader tropism and enhanced infectivity. Therefore, cell-in-cell infection may represent a novel pathway for EBV transmission to non-susceptible ECs, a process 
we term as "in-cell infection".

\section{Results}

Cell-in-cell structures formed between B lymphocytes and ECs in NPC tissues

To verify the presence of cell-in-cell structures in EBV-related NPCs, we first determined the existence of heterotypic cell-in-cell structures in NPC tissues. Indeed, we found that cell-in-cell structures were present in almost all clinical samples by hematoxylin-eosin staining (Figure 1A). The frequencies varied among subjects and NPC tissues (including nonkeratinizing differentiated nasopharyngeal carcinoma (NDNC) and nonkeratinizing undifferentiated nasopharyngeal carcinoma (NUNC); Figure 1B). Based on immunofluorescence staining, heterotypic cell-in-cell structures were characterized by the appearance of $\mathrm{CD}_{20} 0^{+} \mathrm{B}$ cells inside E-cadherin ${ }^{+}$ ECs (Figure 1C). Similar results were obtained with the determination of B cells by CD19 expression (data not shown). This was further confirmed by an independent study with samples from a different hospital (Wang S, data not shown).

We next examined the presence of EBV-encoded early RNA $(E B E R)$ in heterotypic cell-in-cell structures formed between infiltrating lymphocytes and nasopharyngeal ECs using RNA in situ hybridization (ISH) followed by hematoxylin staining. Both EBER-positive and -negative lymphocytes were detected in heterotypic cell-in-cell structures (Figure 1D). We also observed EBER-positive epithelial tumor cells. Most of the heterotypic cellin-cell structures were formed between $E B E R$-negative lymphocytes and $E B E R$-positive epithelial tumor cells (Figure 1E, red bar) or EBER-positive lymphocytes and $E B E R$-negative epithelial tumor cells (Figure 1E, green bar). We also confirmed the presence of heterotypic cellin-cell structures in NPC tissues using transmission electron microscopy (TEM). In a typical cell-in-cell structure, one lymphocyte (indicated by the white arrow) inside one host EC was surrounded by vacuole membrane and the deformed nucleus (indicated by "N") of the host cell (Figure 1F). The internalized lymphocyte underwent cell death inside the vacuole with deformed nucleus and scattered nucleolus, similar to the fate of most effector cells inside host cells [38]. These observations clearly show that heterotypic cell-in-cell structures formed between infiltrating B lymphocytes and ECs are present in vivo in NPC tissues.

EBV-producing Akaka cells transmit virus to EBV CNE2 ECs through cell-in-cell interaction

To directly determine whether EBV-infected B cells could transmit EBV to uninfected ECs by invading into ECs, we co-cultured EBV-negative CNE-2 cells, a human NPC-derived EC cell line without CD21/CR2 receptors [48], with BL-derived EBV-eGFP Akata cells. EBV-eGFP Akata cells were derived from $\mathrm{EBV}^{-} \mathrm{AK} 31$ cell line that was latently infected with recombinant EBV containing an $e G F P$ gene and a neomycin-resistant gene. $e G F P$ gene was inserted into the viral $B X L F 1$ open reading frame to monitor the activation of $\mathrm{EBV}$ and the infection of ECs [47]. This cell line was referred to as GFP-Akata cells afterward. Consistent with the observations in clinical samples described above, both GFP-Akata cells and parent AK31 cells formed heterotypic cellin-cell structures with CNE-2 cells after the co-culture in vitro. Remarkably, GFP-Akata cells formed cell-in-cell structures in higher frequency compared with uninfected parent AK31 cells in parallel (Supplementary information, Figure S1A). To determine whether GFP-Akata cells could transfer EBV to CNE-2 cells through the cell-in-cell mechanism, we used time-lapse microscope to track the invading processes of GFP-Akata cells into CNE-2 cells. We observed the gradual increase of green fluorescence in the internalized GFP-Akata cell $6 \mathrm{~h}$ after the initiation of co-culture, indicating the activation of EBV. The GFP intensity and the percentage of internalized $\mathrm{GFP}^{+}$Akata cells continued to increase until $10 \mathrm{~h}$ (Figure 2A). However, free GFP-Akata cells maintained the similar GFP positivity during co-culture (data not shown). Internalized GFP-Akata cells appeared intact during the observation period (Figure $2 \mathrm{~A}$ ). We individually tracked 135 cell-in-cell structures with GFP $^{-}$ GFP-Akata cells at the beginning of the observation for $12 \mathrm{~h}$. Fifteen internalized cells turned green over the interval (Figure 2B). Among them, three went out of host cells intactly (Figure 2B and Supplementary information, Figure S1C). Most internalized GFP-Akata cells displayed characteristics of entotic cell death (lysotracker positive and caspase-3 negative; Supplementary information, Figure S1B). No green fluorescence was observed in CNE-2 cells without cell-in-cell structures.

By 12 h, GFP-Akata cells expressing GFP also co-expressed ZEBRA protein, which is encoded by viral lytic replication gene BZLF1 (Figure 2C), indicating the productive replication of viral genome in the invading GFP-Akata cells. Considering that the appearance of GFP is the reporter for EBV activation, the EBV activation process in cell-in-cell structures likely depends neither on the conventional activation signals such as IgG ligation in EBV-infected B cells [49] nor on cell-cell contact-dependent activation [50].

When we extended the observation time, the green fluorescence became visible in host CNE-2 cells (Figure 2D 

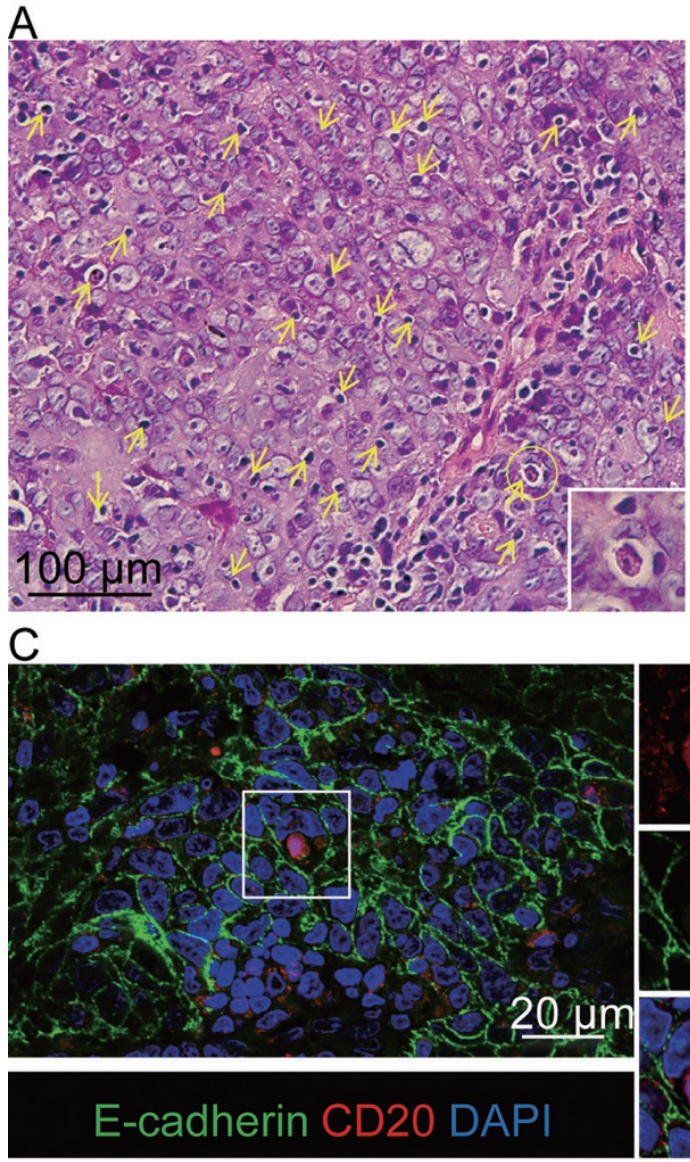

E

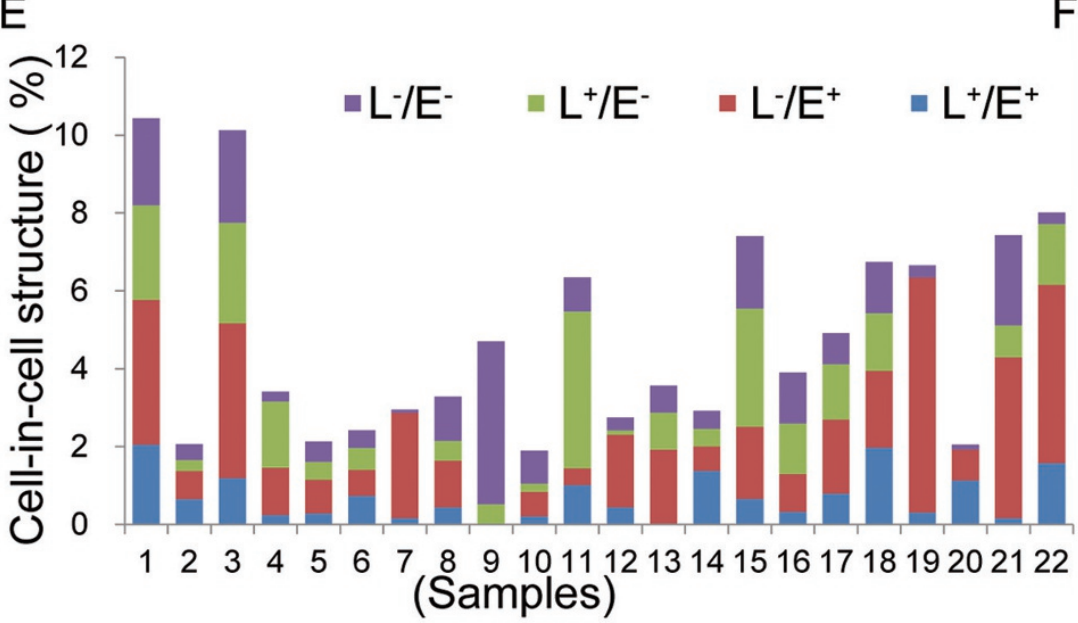

B

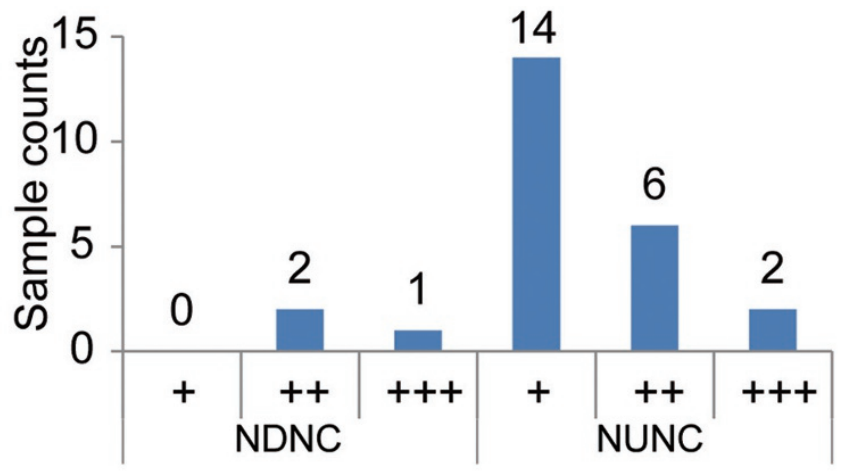

D EBER staining

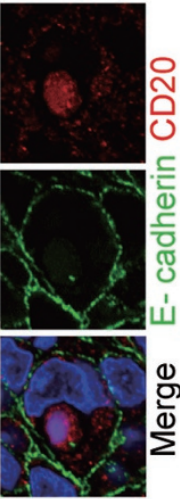

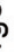

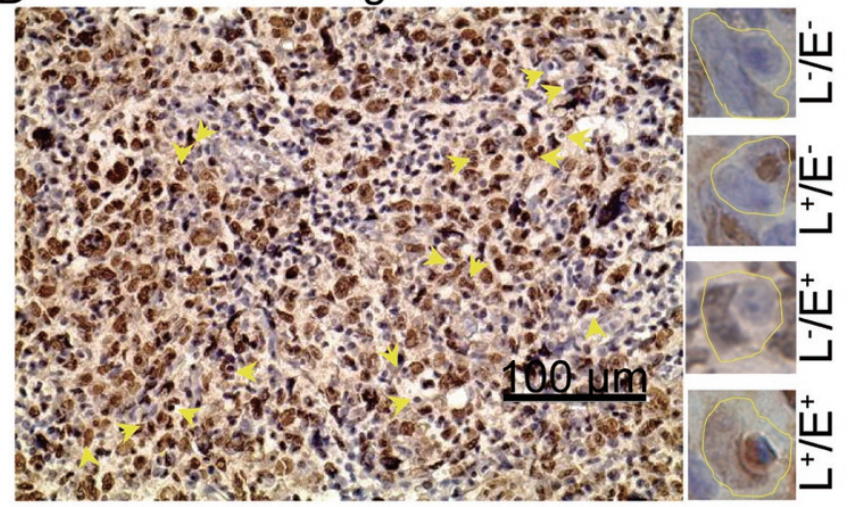

$\mathrm{F}$

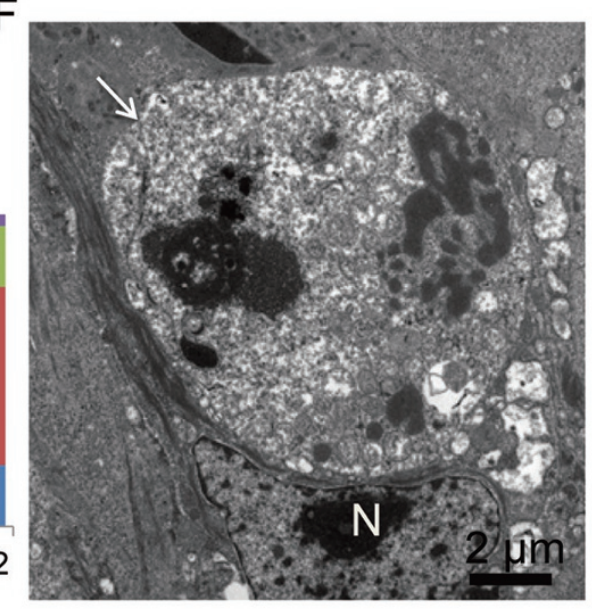

Figure 1 Cell-in-cell structures formed between B lymphocytes and nasopharyngeal ECs in NPC tissues. (A) Typical heterotypic cell-in-cell structures in one NPC tissue sample. Heterotypic cell-in-cell structures were indicated by yellow arrows. (B) Frequency of heterotypic cell-in-cell structures in NDNC $(n=3)$ and type $2 \mathrm{~b}$ (III) NUNC $(n=22)$ determined by hematoxylin-eosin staining. The cell-in-cell frequency was scored with four scales: "-", 0\%; "+”, 1\%-5\%; "++", 5\%-10\%; "+++", 10\%$15 \%$. (C) Representative images of lymphocyte-nasopharyngeal EC cell-in-cell structures in a human NPC sample with co-staining of E-cadherin (green) and CD20 (red). DAPI staining (blue) indicated the nucleus. (D) EBER staining of NPC samples. Four types of heterotypic cell-in-cell structures were presented in the right lane. $\mathrm{L}^{-} / \mathrm{E}^{-}: E B E R^{-}$lymphocytes/EBER ECs; $\mathrm{L}^{+} / \mathrm{E}^{-}:$EBER $R^{+}$lymphocytes/EBER ECs; $\mathrm{L}^{-} / \mathrm{E}^{+}: E B E R^{-}$lymphocytes/EBER $R^{+} \mathrm{ECs} ; \mathrm{L}^{+} / \mathrm{E}^{+}:$EBER $R^{+}$lymphocytes/EBER ECs. (E) Statistics of four types of heterotypic cell-in-cell structures in individual specimen indicated by different colors. (F) $A$ representative TEM image of heterotypic cell-in-cell structure in tissue sections of NPC. In a typical cell-in-cell structure, the internalized B cell (indicated by a white arrow) was surrounded by the vacuole and the deformed nucleus (N) of the EC. 
A
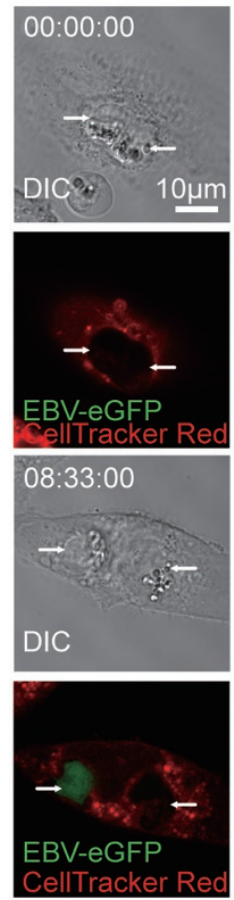

D
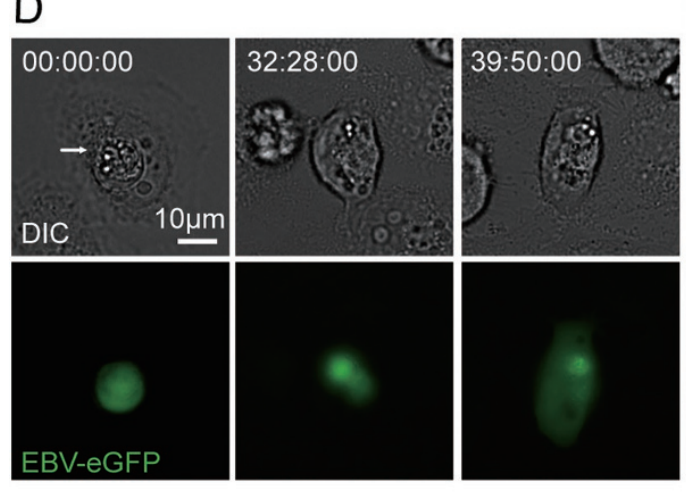

B
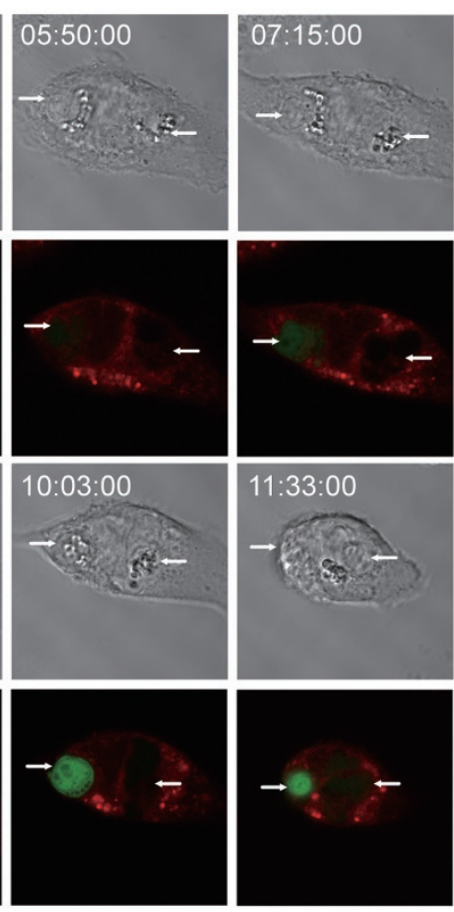

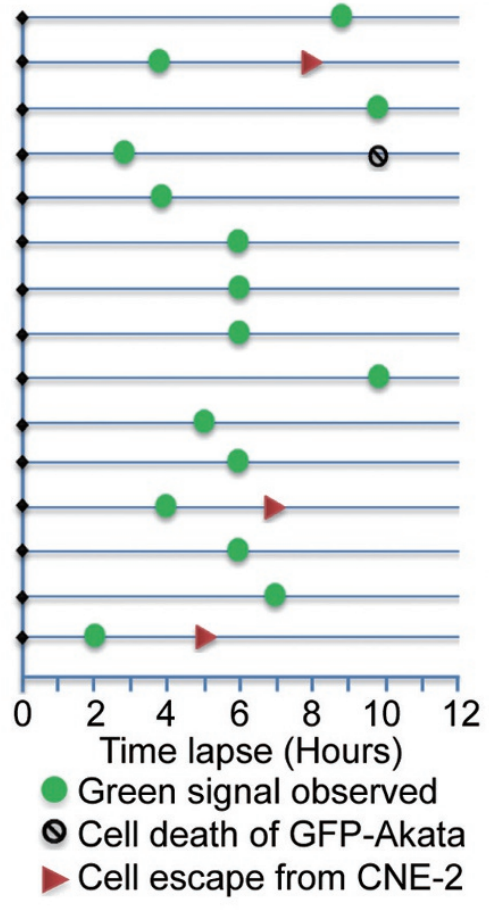

C

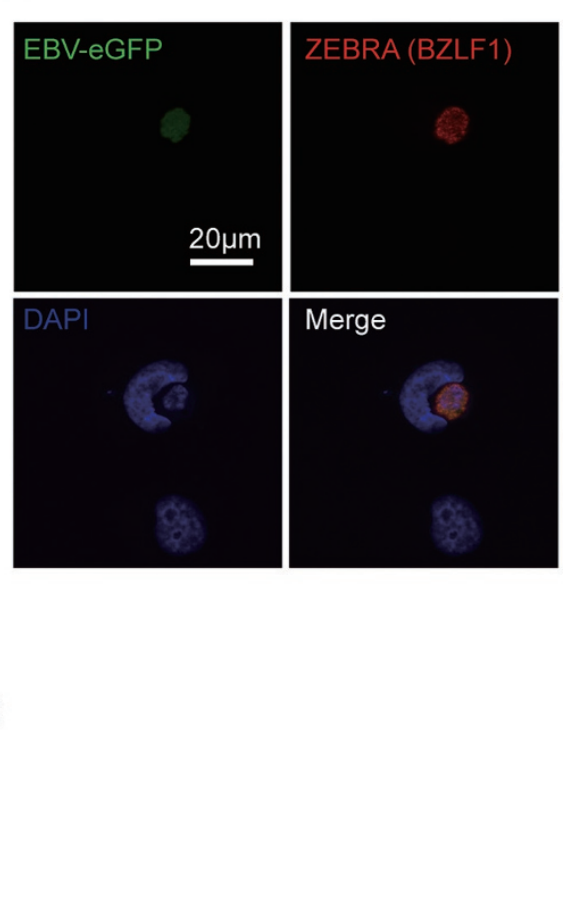

E

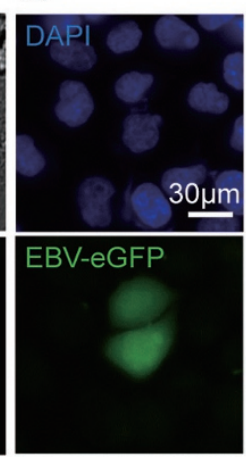

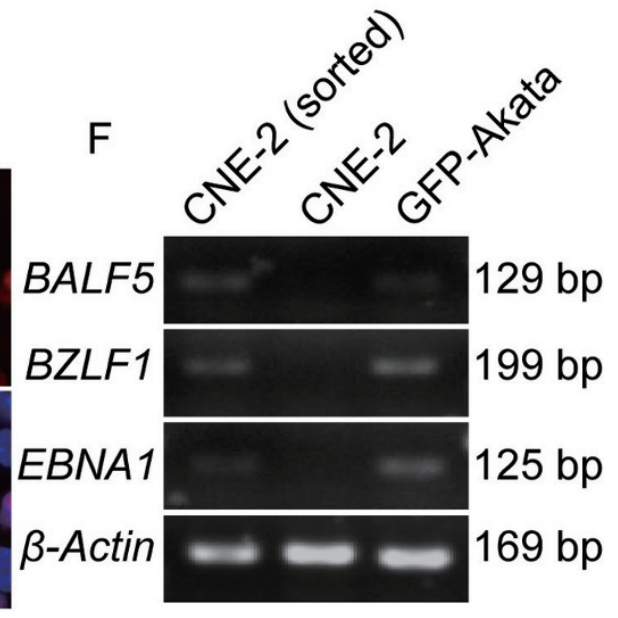

G

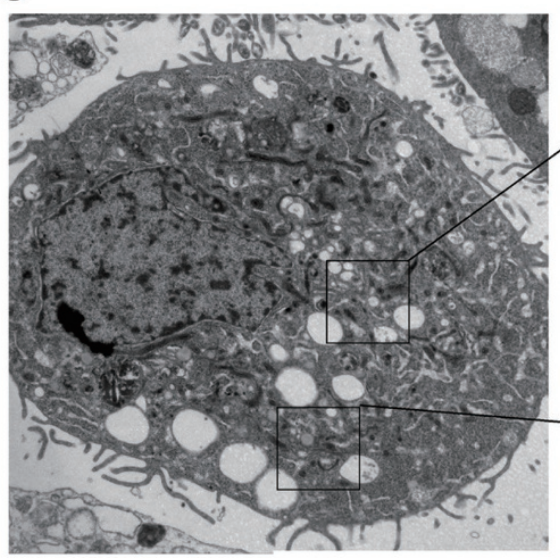

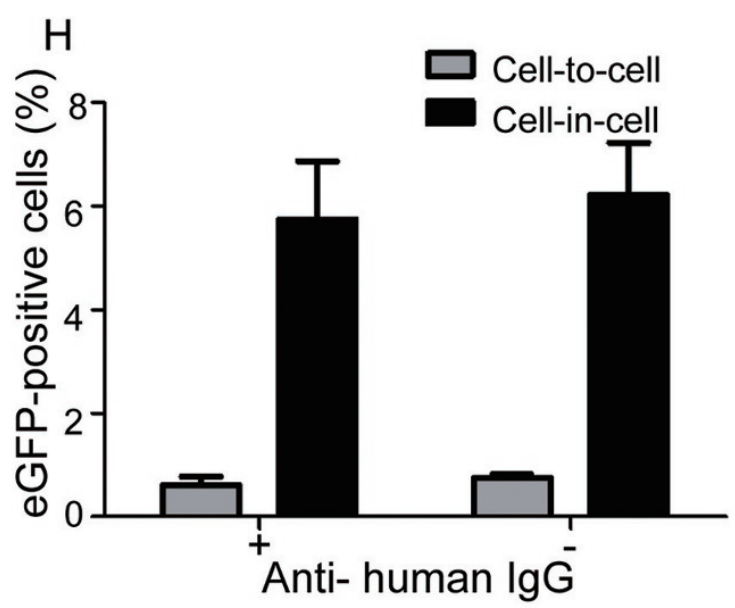


and Supplementary information, Figure S2A). To ensure that EBV can be transmitted to CNE-2 cells through cellin-cell structure formation, GFP-Akata and CNE-2 cells were labeled with CellTracker Green and CellTracker Red dyes, respectively, and co-cultured for $4 \mathrm{~h}$. Double-labeled cells (with the formation of cell-in-cell structures) were sorted by flow cytometry and further selected with G418 for EBV-eGFP-transferred CNE-2 cells with the advantage of neomycin-resistant gene in GFP-Akata cells. Seven days later, $75 \%$ of the sorted CNE-2 cells highly expressed GFP compared with the original CNE2 cells infected with cell-free virus $(<0.1 \%)$. We also probed EBV-specfic EBER to localize EBV in the sorted CNE-2 cells with cell-in-cell structures. EBV was located in the nucleus of the CNE-2 cell (Figure 2E). We further examined the expression of key viral genes and proteins, such as EBNA1 (a gene marker for the latency), $B A L F 5$ (a gene expressing DNA polymerase, a lytic protein) and viral transcriptional regulatory gene BZLF1 (an indicator for lysis induction) of EBV [51], in the sorted CNE-2 cells. As revealed by RT-PCR (Figure 2F) and western blotting assays (Supplementary information, Figure S2B), EBNA1, BALF5 and BZLF1 mRNAs were expressed in the sorted CNE-2 cells with the similar patterns to those of GFP-Akata cells, but not detected in original CNE-2 cells. The accumulation of EBV virions in CNE-2 cells was also detected by TEM (Figure 2G). EBV-like virions were readily observed in the cytoplasm of CNE-2 cells with cell-in-cell structures. The size of the virions was $\sim 180 \mathrm{~nm}$. Most virions contained envelopped capsids (Figure 2G, yellow circle).
Transmission of EBV to ECs through cell-in-cell structures is independent of cell-to-cell contact

Our results strongly support the hypothesis that the cell-in-cell structure is able to mediate virus transmission to non-susceptible ECs. However, we cannot exclude the possibility that the cell-to-cell mechanism also contributes to the transmission of EBV to ECs during co-culture. Therefore, we compared the cell-in-cell-mediated pathway with conventional cell-to-cell-mediated pathway. According to the previously reported cell-to-cell transmission experiments [50], GFP-Akata cells were co-cultured with CNE-2 cells at 5:1 ratio for $24 \mathrm{~h}$ in the presence or absence of goat anti-human IgG polyclonal antibody. Treatment of anti-IgG induced the entry of the viral lytic cycle in GFP-Akata cells. The non-adherent GFP-Akata cells were removed. Fresh medium was added and cells were cultured for another $24 \mathrm{~h}$. We measured the frequencies of $\mathrm{GFP}^{+} \mathrm{CNE}-2$ cells free in the culture and with cell-in-cell structures, which reprensent the cellto-cell and cell-in-cell transmission, respectively. The precentage of free $\mathrm{GFP}^{+} \mathrm{CNE}-2$ cells ranged from $0 \%$ to $1 \%$ with the average of $0.67 \%$ in total free CNE- 2 cells. Upon the treatment of anti-IgG, the infection rate slightly increased to $0.81 \%$. However, almost $6 \%$ of CNE-2 cells with cell-in-cell structures became infected (as evidenced by the appreance of GFP fluorescence) within 48 $\mathrm{h}$ regardless of the anti-IgG treatment (Figure $2 \mathrm{H}$ ). These results suggest that cell-in-cell-mediated EBV transmission to non-susceptible ECs in our experiments occurred independently of cell-to-cell contact.

These data thus strongly suggest that cell-in-cell struc-

Figure 2 EBV-carrying GFP-Akaka cells transmit virus to CNE-2 cells through cell-in-cell interaction. (A) Time tracking observation of EBV activation in GFP-Akata cells within CNE-2 cells. CNE-2 cells (pre-stained with CellTracker Red dye) were co-cultured with GFP-Akata cells at a ratio of 1:10 and observed using LSM 710 confocal microscope. It was notable that internalized GFP-Akata cells became green (GFP-positive), which indicated EBV activation, at time 07:15:00. Time was indicated as hour:minute:second. Scale bar,10 $\mu \mathrm{m}$. (B) The fate of individual GFP-Akata cell was indicated, including the appearance of GFP (green circle), undergoing cell-in-cell death (black circle) or escaping from CNE-2 cells (red triangle). Data analysis was performed for $12 \mathrm{~h}$ with an $1 \mathrm{~h}$ interval. (C) Expression of ZEBRA (red) in an EBV-activated GFP-Akata cell (green) inside a CNE-2 cell (indicated by DAPI) as determined by immunofluorescence staining. (D) Time tracking analysis of GFP diffusion into CNE-2 cells. The CNE-2 cells were co-cultured with GFP-Akata cells at a ratio of 1:5 and observed using DMI6000B fluorescence microscope. The target CNE-2 cells became GFP positive at time 39:50:00, which was the indicator of EBV infection. Time was indicated as hour:minute:second. Scale bar, $10 \mu \mathrm{m}$. (E) Distribution of EBER (red) and GFP (green) in cell-in-cell structures. After incubation of GFP-Akata cells with CNE-2 cells for $12 \mathrm{~h}$, the free GFP-Akata cells were removed by washing with PBS. The sorted CNE-2 cells with cell-in-cell structures were hybridized with the probe to EBER for ISH. (F) PCR analysis of the indicated viral mRNAs in the sorted CNE-2 cells with cell-in-cell structures. GFP-Akata cells served as positive control while non-treated CNE-2 cells served as negative control. (G) TEM observation of EBV virions (yellow circle) in in-cell infected CNE-2 cells. The in-cell infected CNE-2 cells were obtained by FACS sorting after co-culturing with GFP-Akata cells at a ratio of 1: 10 and maintained with G418-containing culture medium for 2 weeks. (H) A summary of cell-in-cell- and cell-to-cell-mediated EBV transmission. CNE-2 cells were co-cultured with GFP-Akata cells (with or without anti-human IgG treatment) for $24 \mathrm{~h}$. The frequencies of GFP-positive CNE-2 cells freely or with cell-in-cell structures were analyzed by fluorescence microscopy. 
ture formation between GFP-Akata B cells and CNE-2 cells not only promotes the activation of EBV in B cells, but also facilitates the spreading of EBV from EBV-infected B cells to ECs. The formation of cell-in-cell structures might be an alternative mechanism of virus tansmission to non-susceptible ECs, which we term as "in-cell infection".

EBV from in-cell infected CNE-2 cells possesses enhanced infection potency and altered tropism

Our investigation revealed a novel pathway of EBV transmission from B cells to ECs through a cell-in-cell process, which enables ECs to express viral proteins and to package viral particles. The next question is whether these ECs are able to secrete infectious virions. Taking advantage of the neomycin-resistant gene in GFP-Akata cells, we successfully obtained CNE-2 cells expressing EBV viral protein EBNA1 (Supplementary information, Figure S2B) after cell-in-cell structure formation. To distinguish original CNE-2 cells and the sorted in-cell infected CNE-2 cells, we referred to the latter as iCNE-2 cells.

We stimulated GFP-Akata and iCNE-2 cells with anti-human IgG [49] and sodium butyrate [52], respectively, to initiate the EBV virion production. After stimulation for 3 days, culture supernatants containing EBV virions were collected. To distinguish EBV virions derived from these two types of cells, A-EBV and C-EBV were named for EBVs from GFP-Akata and iCNE-2 cells, respectively. By using the same amount of virions normalized by quantitative PCR, we first performed the cellfree infection assay in susceptible AK31 and non-susceptible CNE-2 cells to compare the infectivity between A-EBV and C-EBV. A-EBV maintained the capacity to infect AK31 cells at the percentage of $\sim 10 \%$ but could not infect CNE-2 cells (Figure 3A left and right, blue line for A-EBV infection). Surprisingly, based on the expression of GFP fluorescence, we found that C-EBV from iCNE-2 cells infected both AK31 (Figure 3A, left, red line, $10.1 \%$ ) and CNE-2 cells (Figure $3 \mathrm{~A}$, right, red line, $14.8 \%$ ). It is well established that deficiency of EBV receptor such as CD21 molecules on ECs is the main obstacle for their susceptibility to cell-free EBV infection [19]. However, the extra ability of C-EBV to infect CNE2 cells suggests that CNE- 2 cells infected via in-cell infection are able to assemble virions with an extra capacity to infect non-susceptible ECs. This was confirmed when we further compared the capacity of A-EBV and $\mathrm{C}$-EBV to infect other lymphocytes or EC-derived cell lines (Figure 3B). While A-EBV only infected B cell line AK31, C-EBV infected both lymphocytes (AK31) and EC cell lines (i.e., CNE-2, A431, MCF-7, PLC/PRF/5 and an immortalized epithelial cell line NPEC1-Bmil) [53].

EBV attaches to B cells and ECs in different ways. To understand the extended tropism of EBV gained from in-cell infection, we further performed the adsorption experiments to determine the attachment and subsequent infectivity of A-EBV and C-EBV (Supplementary information, Figure S3). A-EBV and C-EBV were incubated with both AK31 and CNE-2 cells for $2 \mathrm{~h}$ on ice, the supernatants were collected and fresh media were added to the cells. We calculated the percentages of infected AK31 and CNE-2 cells based on the appearance of GFP. The collected culture supernatants containing free viruses were added to fresh AK31 and CNE-2 cells. The infection rates were compared. As indicated in Figure 3C, A-EBV only infected AK31 cells with the infection rate of $10 \%-15 \%$, and the unbound A-EBV collected from the AK31 absorption assay showed decreased infection rate for AK31 cells (1\%-5\%). A-EBV did not infect $\mathrm{CNE}-2$ cells. No $\mathrm{GFP}^{+} \mathrm{CNE}-2$ cells were detected after the incubation with A-EBV. Unbound A-EBV from the CNE-2 cell absorption assay could continue to infect AK31 cells with an infection rate (8\%-12\%) comparable to that of original A-EBV (10\%-15\%; Figure 3C). On the other hand, C-EBV retained the ability to infect AK31 cells and gained an extra ability to infect CNE-2 cells (Figure 3D). C-EBV infected AK31 with the infection rate of $12 \%-15 \%$, which was comparable to that of A-EBV (10\%-15\%). In addition, C-EBV infected CNE2 cells with $5 \%-10 \%$ infection rate, much higher than that of A-EBV $(<0.1 \%)$. Interestingly, unbound C-EBV collected from the AK31 absorption assay could infect both AK31 and CNE-2 cells with similar infection rates, like the unbound C-EBV from CNE-2 cell supernatants (Figure 3D).

C-EBV could also infect other B cells through cellto-cell infection. When co-cultured with iCNE-2 cells, $58.5 \%$ of Raji cells were GFP positive, which was comparable to AK31 cells (76.8\%; Figure 3E). These results suggest a mixed property of C-EBV generated from iCNE-2 cells, enabling C-EBV to infect both B cells and ECs. Our results thus demonstrate that compared with GFP-Akata B cell-derived EBV virions, EBV virions from in-cell infected iCNE-2 cells display improved infection potency and altered tropism.

\section{In-cell infection occurs in normal ECs}

We have demonstrated in vitro that EBV-loaded GFP-Akata B cells could invade NPC cell line CNE2, leading to the transmission of EBV to host CNE-2 cells. However, tumor cells are not equivalent to normal ECs. Therefore, we investigated the occurrence of in- 

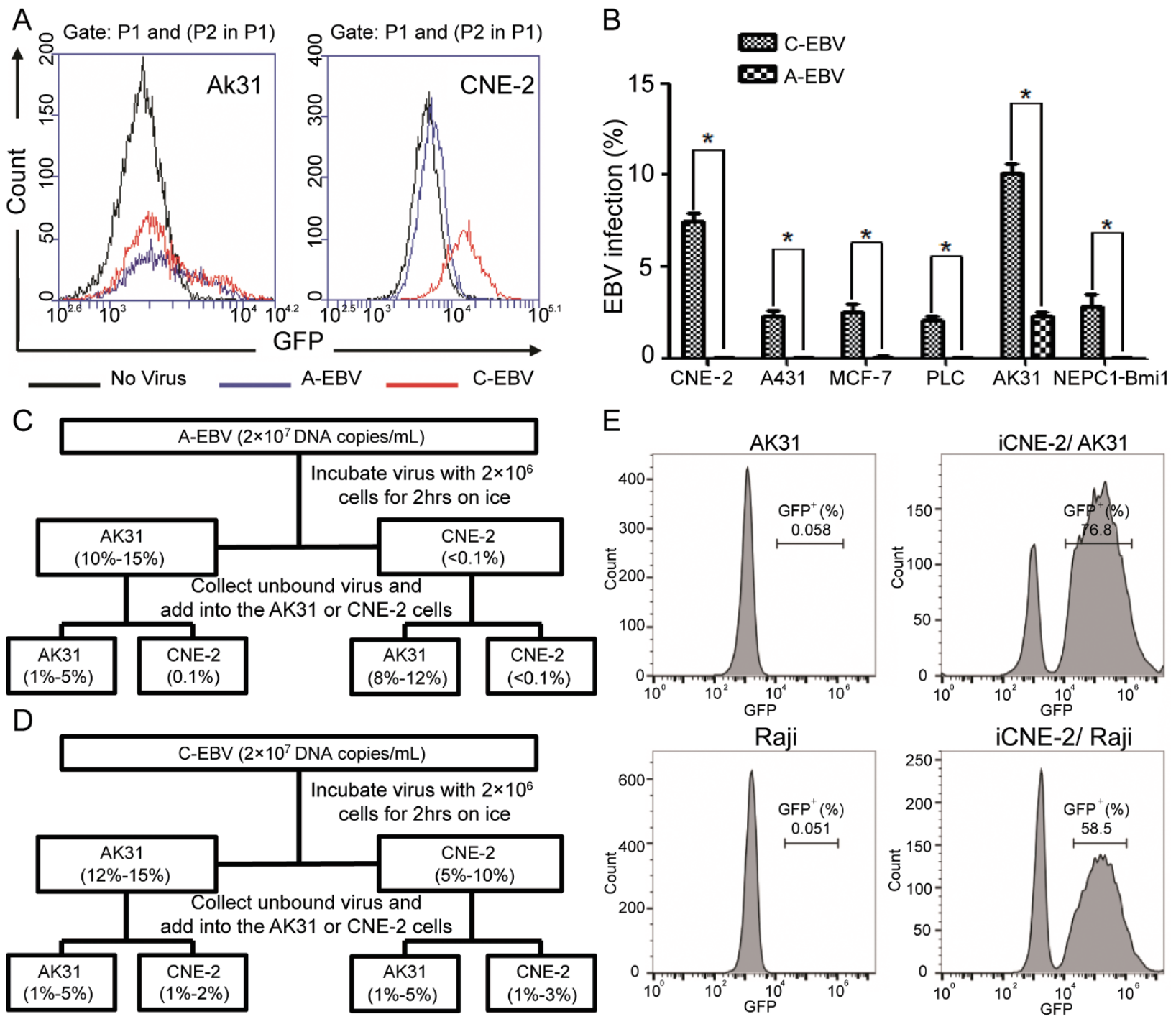

Figure 3 EBV from in-cell infected CNE-2 cells possesses enhanced infection potency and altered tropism. (A) Determination of GFP ${ }^{+}$AK31 cells (left) and CNE-2 cells (right) after the infection with A-EBV from GFP-Akata cells (blue line) or C-EBV from iCNE-2 cells (red line) for $24 \mathrm{~h}$ by flow cytometry. Cells without EBV treatment served as negative control (black line). (B) Statistical comparison of percentages of CNE-2, A431, MCF-7, PLC/PRF/5, AK31 and NEPC1-Bmi1 cells with GFP expression after $24 \mathrm{~h}$ cell-free infection with A-EBV or C-EBV by immunofluorescence microscopy. All experiments were repeated at least three times. ${ }^{*} P<0.05$. (C, D) Absorption assay of A-EBV (C) or C-EBV (D) was performed. $2 \times 10^{7}$ DNA copies $/ \mathrm{ml}$ A-EBV (C) or C-EBV (D) virus was incubated with $2 \times 10^{6}$ AK31 or CNE-2 cells for $2 \mathrm{~h}$ on ice. Supernatants were collected and the fresh medium was added to cells for culture. The percentage of GFP ${ }^{+}$AK31 or CNE-2 cells was determined by flow cytometry after $12 \mathrm{~h}$. The collected supernatants were added to fresh AK31 and CNE-2 cells to determine the infection rate after $12 \mathrm{~h}$. (E) AK31 or Raji cells were co-cultured with in-cell infected iCNE-2 cells for $24 \mathrm{~h}$. The expression of GFP in AK31 (upper) or Raji (lower) cells was analyzed by flow cytometry. AK31 and Raji cells only (left) served as negative controls.

cell infection in normal ECs by using an immortalized epithelial cell line NEPC1-Bmi1 [53] as the host cell. NEPC1-Bmil cells formed heterotypic cell-in-cell structures with GFP-Akata cells in a higher frequency than with AK31 cells, which reached $1 \%$ for GFP-Akata cells and $0.66 \%$ for Ak31 cells at $24 \mathrm{~h}$ (Figure 4A). However, the percentage of NEPC1-Bmi1 cells with cell-in- cell structures was lower than that of CNE-2 cells. GFP GFP-Akata cells inside NEPC1-Bmil cells were detected $24 \mathrm{~h}$ after co-culture (Figure 4B). At $48 \mathrm{~h}$, green fluorescence was observed in NEPC1-Bmil cells with cell-incell structures (Figure 4C). This indicates that NEPC1Bmil cells can be infected by EBV through cell-in-cell pathway. EBV-derived LMP2A and EBNA1 were co-lo- 


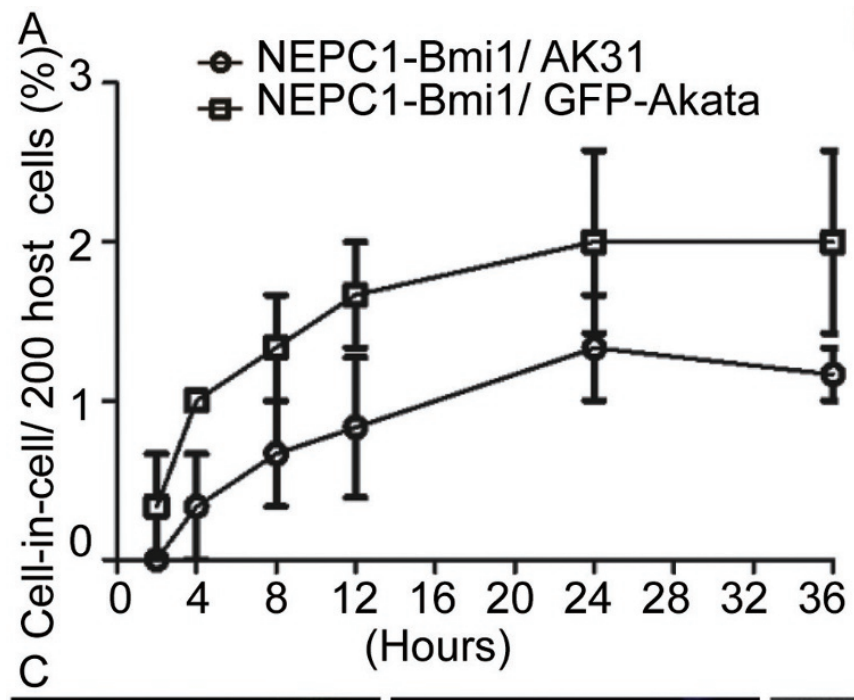

B
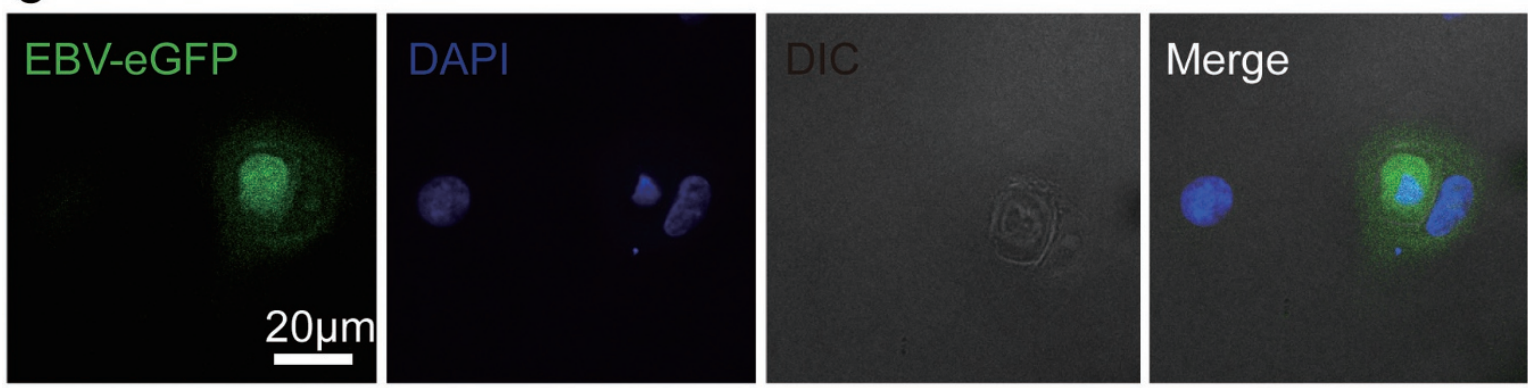

D
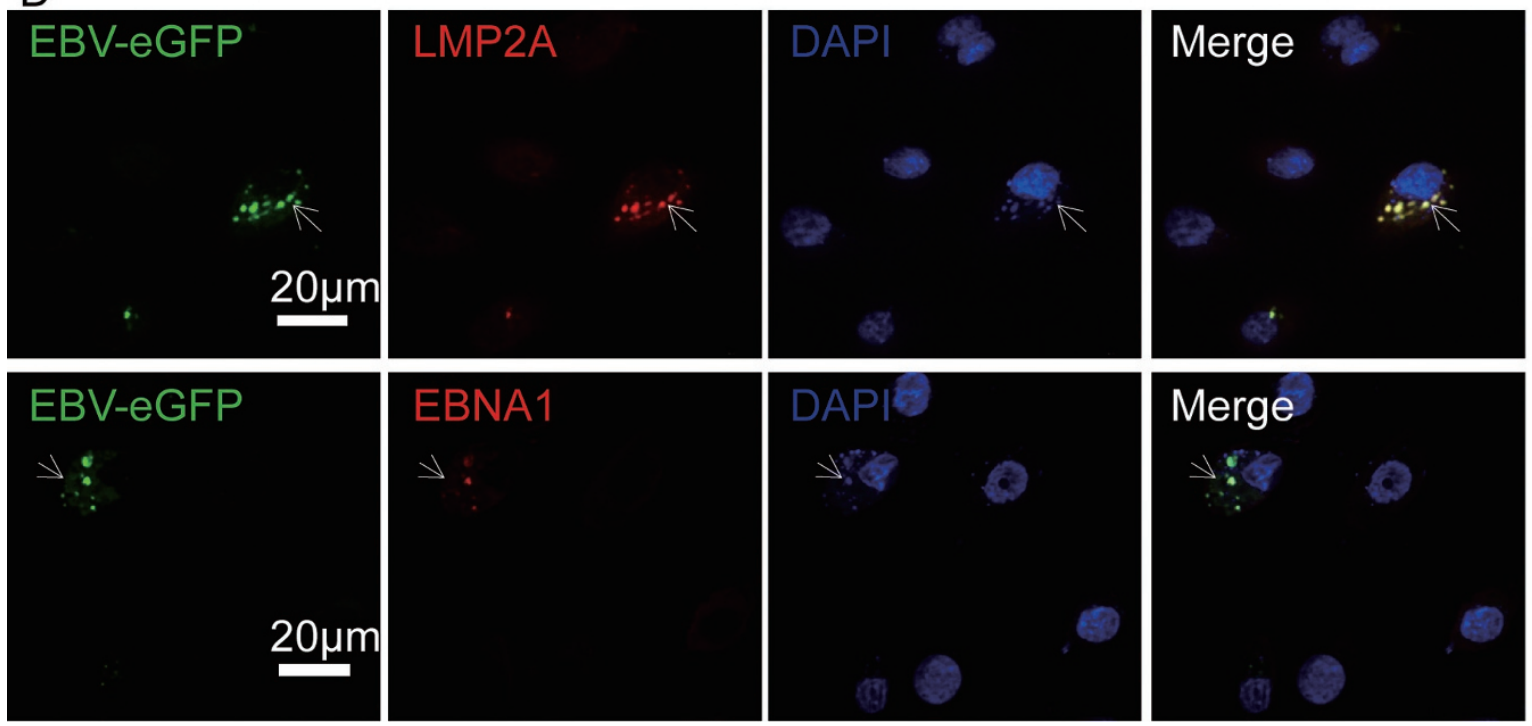

Figure 4 In-cell infection occurs in normal ECs. (A) Percentage kinetics of cell-in-cell structure formation between NEPC1Bmi1 cells and GFP-Akata or AK31 cells. The NEPC1-Bmi1 cells were co-cultured with GFP-Akata or AK31 cells at a ratio of 1:10. The frequencies of cell-in-cell structures were counted at the indicated times. (B) Representative images of EBV activation in an internalized GFP-Akata cells within an NEPC1-Bmi1 cell. The NEPC1-Bmi1 cells were co-cultured with GFP-Akata cells at a ratio of 1:10 for $24 \mathrm{~h}$, followed by fixation and DAPI staining. GFP fluorescence was distributed in internalized GFP-Akata cells. (C) Representative images of the appearance of GFP fluorescence in an NEPC1-Bmi1 cell with an internalized GFP ${ }^{+}$GFP-Akata cell. The NEPC1-Bmi1 cells were co-cultured with GFP-Akata cells at a ratio of 1:10 for $48 \mathrm{~h}$, followed by fixation and DAPI staining. GFP fluorescence was distributed in both internalized GFP-Akata cells and NEPC1Bmi1 cells. (D) Co-localization of GFP fluorescence with LMP2A (upper panel) or EBNA1 (lower panel) in NEPC1-Bmi1 cells. The NEPC1-Bmi1 cells were co-cultured with GFP-Akata cells at a ratio of 1:10 for $24 \mathrm{~h}$, followed by LMP2A or EBNA1 immunofluorescence staining and subsequent DAPI staining. 
calized with the GFP protein (Figure 4D), which further verified the successful infection of NEPC1-Bmil cells through cell-in-cell pathway. We compared the abilities of C-EBV and A-EBV to infect NEPC1-Bmil cells. Similar to CNE-2 cells, C-EBV infected NEPC1-Bmil cells at a higher frequency than A-EBV (Figure 3B). These results demonstrate that like tumor-derived ECs, normal ECs can also undergo in-cell infection by EBV-infected B cells.

Activation of EBV inside CNE-2 cells depends on the PI3K/AKT signaling pathway

Activation of EBV in infected $B$ cells by anti-IgG treatment has been well documented [49]. B-cell receptor (BCR) ligation triggers the activation of the $\mathrm{Src}$ tyrosine kinase Lyn and the downstream PI3K/AKT signaling pathway. This activates the transcription of $B Z L F 1$, which is a switch for EBV activation from latent status. In turn, BZLF1-encoding protein ZEBRA initiates the replication of virus [54]. We found that EBV in GFP-Akata cells was activated autonomously inside host CNE-2 cells in the absence of extrinsic stimuli. It is evident that the PI3K/AKT pathway is key to EBV activation in both cell-free and cell-to-cell contact infections $[50,55]$. We then asked whether the PI3K/AKT signaling pathway was turned on during the autonomous EBV activation after cell-in-cell structure formation. We detected the phosphorylation of $\mathrm{AKT}^{\mathrm{Ser} 473}$ in GFP-Akata cells with cell-in-cell structures. By $12 \mathrm{~h}$, the GFP fluorescence was co-localized with phosphorylated AKT in GFP-Akata cells, but was not detected in CNE-2 cells (Figure 5A). Similarly, no phosphorylation of AKT was observed in AK31 cells when they entered CNE-2 cells during the same observation time (Supplementary information, Figure S4). Our results were in accordance with the report [55] that EBV immediate-early protein BRLF1 could induce the activation of PI3K/AKT and the subsequent viral activation. This suggests the involvement of the PI3K/ AKT signaling in EBV activation within GFP-Akata cells during in-cell infection. When the co-culture continued, the level of phosphorylated AKT increased in $\mathrm{GFP}^{+}$ GFP-Akata cells inside CNE-2 cells (Figure 5B). To further demonstrate the role of the PI3K/AKT signaling in EBV activation, the broad-spectrum PI3K signaling inhibitor, Wortmannin, was added after GFP-Akata cells were co-cultured with CNE-2 cells for 2 or $4 \mathrm{~h}$. After another $24 \mathrm{~h}$ co-culture, GFP signal and AKT phosphorylation were measured in cell-in-cell structures. As shown in Figure 5C, Wortmannin dramatically inhibited the phosphorylation of AKT inside GFP-Akata cells (Figure $5 \mathrm{C}$, left). In parallel, GFP expression in GFP-Akata cells inside CNE- 2 cells also decreased dramatically (Figure
$5 \mathrm{C}$, right). We obtained the similar results when we used another PI3K inhibitor LY296002 (data not shown). These results suggest that the PI3K/AKT signaling is involved in EBV activation within B cells after cell-in-cell structure formation.

\section{Discussion}

In the present study, we describe a novel mechanism of EBV transmission to non-susceptible ECs. We verified that heterotypic cell-in-cell structure formation mediated the efficient transmission of EBV from infected B cells to uninfected non-susceptible ECs, which we call "incell infection". More importantly, EBV virions generated from in-cell infected ECs exhibited excess ability to infect both B cells and ECs, indicating the extension of viral tropism through in-cell infection.

Viral tropism determines the target cells or tissues during infection $[9,10]$. Viruses in most cases infect tropic cells in a receptor-mediated manner [20]. However, certain alternative infection mechanisms have been reported [30]. During EBV infection, it is well accepted that due to the lack of EBV receptors on ECs, cell-tocell infection is the main pathway for EBV infection rather than receptor-mediated cell-free infection [31, 32]. During cell-to-cell infection, the transmission of EBV from $\mathrm{B}$ cells to ECs relies on the conjugate formation between B cells and ECs, which facilitates the entry of viruses into ECs through either membrane fusion or endocytosis [32]. Compared with cell-to-cell infection, in-cell infection undergoes an alternative infection pathway in which its tropism rarely relies on the interaction between host cells and virus and the transmission occurs more rapidly and efficiently. It has been reported that successful cell-to-cell infection of EBV happened after $24 \mathrm{~h}$ [50]. However, in-cell infection initiated within $4 \mathrm{~h}$ with the rapid formation of cell-in-cell structures. EBV was subsequently activated by $6 \mathrm{~h}$ (Figure 2B and Supplementary information, Figure S1C) and the activation was sustained. With the occurrence of lysosome-dependent cellin-cell death (entosis) of the internalized EBV-infected GFP-Akata B cells (Supplementary information, Figure S1B), EBV was released into host ECs to accomplish the transmission of viruses. This is in accordance with the observation that the expression of GFP, phosphorylation of $\mathrm{AKT}^{\mathrm{Ser} 473}$ and entosis of GFP-Akata cells occurred in parallel. Unlike the cell-to-cell spread of EBV to polarized oral ECs, which requires the conjugation between $\mathrm{CD} 11 \mathrm{~b}$ on B cells and heparan sulphate moieties of CD44v3 and LEEP-CAM on ECs [32], the successful in-cell infection is expected to depend on molecules involved in cell-in-cell structure formation such as ezrin or 
A
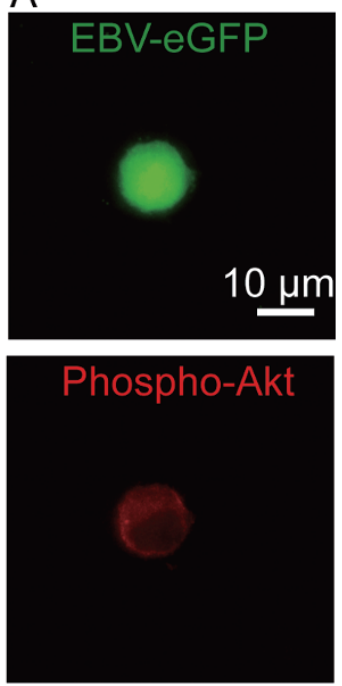

C
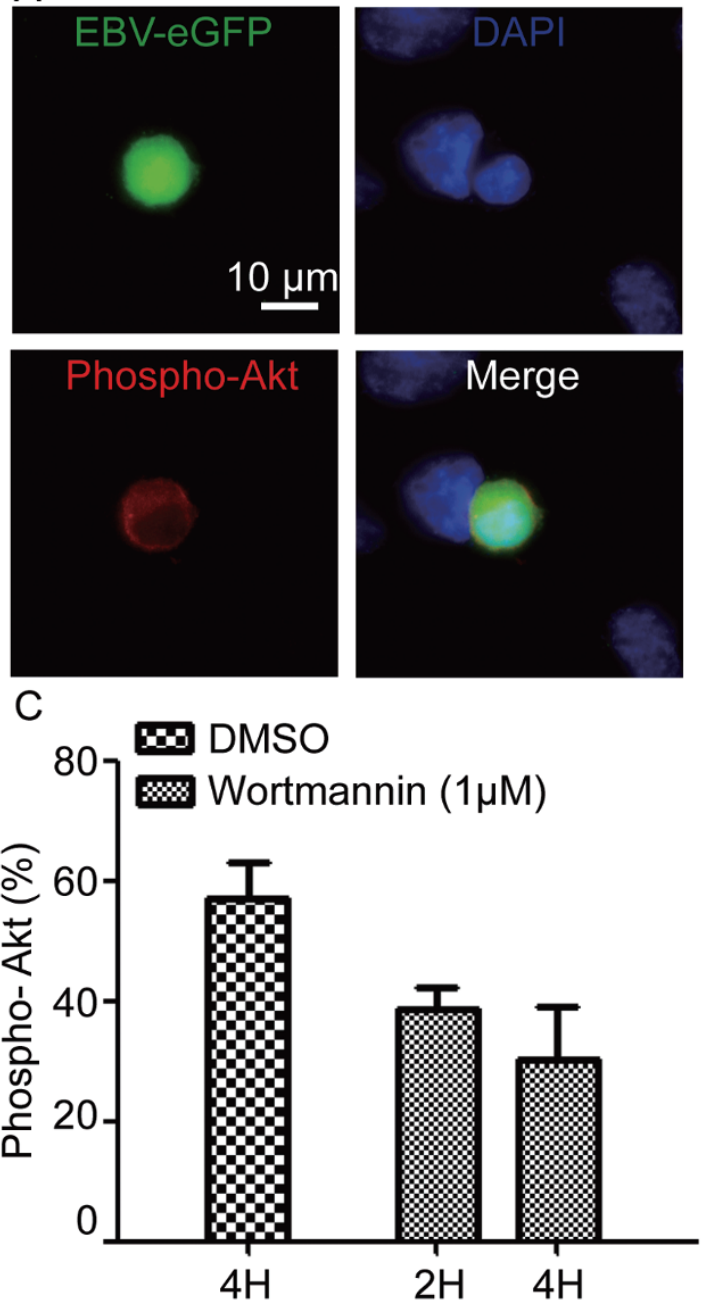

B
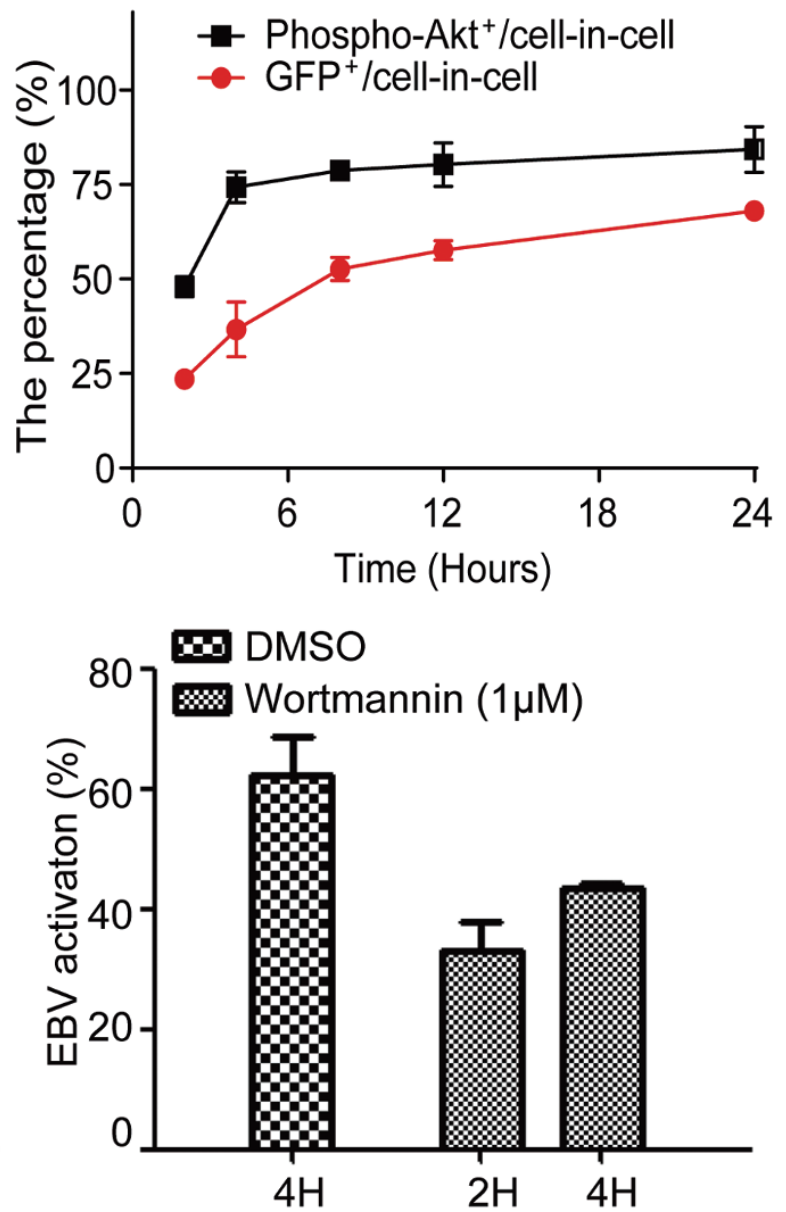

Figure 5 Autonomous activation of EBV inside CNE-2 cells depends on the PI3K/AKT signaling pathway. (A) GFP-Akata cells were co-cultured with CNE-2 cells for $12 \mathrm{~h}$ and the phosphorylation of AKT inside GFP-Akata cells was detected by immunofluorescence staining using anti-phospho-AKT antibody followed by DAPI staining. The images were captured under confocal laser scanning microscope. (B) Proportional kinetics of phospho-AKT ${ }^{+}$GFP-Akata and GFP ${ }^{+}$CNE-2 cells within cell-in-cell structures. GFP-Akata cells were co-cultured with CNE-2 cells. The percentages of phospho-AKT ${ }^{+}$GFP-Akata and GFP ${ }^{+}$CNE-2 cells were analyzed by confocal laser scanning microscopy at the indicated times. The experiments were performed three times independently. (C) The PI3K signaling inhibitor Wortmannin was added to the culture medium after cell-in-cell structure formation (2 or $4 \mathrm{~h}$ after co-culture of GFP-Akata and CNE-2 cells). The percentages of phospho-AKT ${ }^{+}$ GFP-Akata cells (left) and GFP ${ }^{+}$GFP-Akata cells (right) within cell-in-cell structures were determined by fluorescence microscopy $24 \mathrm{~h}$ after the treatment.

ICAM-1 [41, 45]. The exact molecules participating in initiating in-cell infection need to be experimentally verified.

Another interesting issue of in-cell infection is the triggering factor for the EBV activation inside host cells independent of the extrinsic stimuli. It is widely accepted that EBV activation is the prerequisite for the viral infection of ECs either by cell-free virions or through cell-tocell contact $[30,50]$. The molecular mechanism of EBV activation in infected $\mathrm{B}$ cells upon anti-IgG treatment has been well illustrated [49]. BCR ligation triggers the activation of the Src tyrosine kinase Lyn and the downstream PI3K/AKT signaling pathway, which promotes the transcription of $B Z L F 1$ and $B R L F 1$ and subsequently initiates the replication of virus $[54,55]$. The PI3K/AKT pathway has been reported to play crucial roles in cellto-cell EBV transfer as well [50]. We have also demonstrated the phosphorylation of AKT at the early stage 
after cell-in-cell structure formation with the appearance of GFP signals. This suggests the involvement of the $\mathrm{PI} 3 \mathrm{~K} / \mathrm{AKT}$ signaling pathway in the activation of EBV in GFP-Akata cells inside CNE-2 cells. We thus propose that when internalized EBV-infected B cells are wrapped in the vacuole of host cells, certain factors inside the specific microenvironment of the vacuole might dedicate to the activation of EBV. We also found that the activation of AKT occurred simultaneously with the initiation of cell-in-cell entotic death (data not shown). The activation of EBV and the occurrence of entotic death of B cells inside CNE-2 cells thus constitute two prerequisites for the efficient transmission of EBV from internalized GFP-Akata cells to CNE-2 cells. The significance of ligation-independent EBV activation is that the latency of EBV can be rapidly interrupted when EBV-burdened B cells are internalized into ECs. This process might be related to the intrinsic maintenance of long-term persistent infection.

More importantly, our results revealed that in-cell infection altered the tropism of virions. EBV generated from in-cell infected CNE-2 cells exhibited high potency to infect both B cells and ECs in cell-free conditions. It is reported that EBV infection of ECs is supposed to allow the virus to gain optimal tropism [56] and the rapid expansion of infection [33]. During in-cell infection, EBV is activated in tropic B cells and released to ECs after entotic death of B cells inside ECs (Supplementary information, Figure S1B). With the entotic death of B cells, in-cell infected ECs thus possess the cellular components of both B cells and ECs. The cells might assemble EBV virion particles in alternative manners with extended viral tropism and higher efficacy. Another possibility lies in the genetic and epigenetic alterations of EBV after incell infection. This is consistent with the "cell-in-cell selection" model that we suggested before [39], in which the internalized cells adapt to the internal microenvironment to package EBV in a separate process. The exact molecular mechanisms are under investigation.

With the prevalence of heterotypic cell-in-cell structure formation between virus-loaded immune cells and tissue cells during chronic infection [38], in-cell infection of non-susceptible ECs with broader potency and higher efficiency might be more inclined to form the viral latency. The cell-in-cell pathway not only mediates the infection of ECs but also provides virus-bearing tropic cells inside ECs with a shelter to escape from immune attack. A few reports have described the presence of virions in oropharyngeal or nasopharyngeal epithelium $[16,57]$ and the phenomena of latent or replicating EBV in intro-epithelial B cells [46]. However, the exact mechanisms are unclear. According to our study, heterotypic cell-in- cell structure formation in vitro between EBV-infected B cells and ECs to some extent recapitulates the interaction between infiltrating lymphocytes and tissue cells during persistent infection. Our findings thus favor the view that ECs undergoing EBV in-cell infection might be a more stable virus pool in vivo to maintain the latency of EBV owing to the high incidence of heterotypic cell-in-cell structure formation in local inflammatory region. In addition, heterotypic cell-in-cell structure formation between $\mathrm{B}$ cells and ECs might also induce the chromosome instability and formation of aneuploidy, which has been reported during the formation of homotypic cell-in-cell structures [58]. To our opinion, in-cell infection-mediated EBV persistency and heterotypic cell-in-cell structure formation-mediated aneuploidy perform the "double shocks", which may accelerate the transformation of normal ECs and the subsequent nasopharyngeal carcinogenesis.

In recent years, the cell-in-cell structure has aroused broad concerns as increasing evidence has supported its roles in development [59,60], homeostasis [61], aging $[62,63]$, malignant transformation [64] and tumorigenesis [58] across a wide range of species (encompassing lower eukaryotes and mammals), exhibiting pathophysiological significance. More recently, the cell-in-cell structure formation is demonstrated to be able to promote clonal selection and human tumor evolution mediated by cell competition, a mechanism evolved to eliminate less fit cells from a heterogeneous cell population $[65,66]$. In this study, we have demonstrated its involvement in EBV viral infection of non-susceptible ECs through the invasion of B cells into ECs. This will be helpful to explain the observation of the presence of HIV in intestinal and genital ECs in AIDS patients, which has been extensively debated. Considering the diversity of virus-infected cells such as B or T cells and their engagement in cellin-cell structures during chronic infection, in-cell infection might represent a general receptor-independent mechanism of viral infections of non-susceptible tissue cells. The cell-in-cell structure formation might thus participate in the pathogenesis of viral infection through its unique biological behavior. Further investigations on its molecular mechanisms will undoubtedly provide new clues and targets for the prevention and treatment of viral infections in clinic.

\section{Materials and Methods}

Immunohistochemistry and immunofluorescence staining in tissues

The paraffin-embedded specimens from 25 EBV-infected NPC patients were collected from the Cancer Hospital in Guangzhou ( $n$ $=22$ ) and the Second Affiliated Hospital of Guangzhou Medical 
University $(n=3)$. Hematoxilin/eosin (Vector Laboratories, Germany) staining was performed according to the standard protocols to determine the heterotypic cell-in-cell structures in NPC samples. In addtiton, specimens were stained with mouse anti-CD20 antibody (Boster Company, China), rabbit anti-LMP2A polyclonal antibody (Proteintech Group Inc, USA) and mouse anti-human EBNA1 McAb (ab8329; Abcam Company, USA) followed by Alexa Fluor 568-conjugated goat anti-rabbit IgG $(\mathrm{H}+\mathrm{L})$ Antibody and Alexa Fluor 594-conjugated goat anti-mouse IgG $(\mathrm{H}+\mathrm{L})$ secondary antibodies (Invitrogen, USA). Flurenscence signals were observed under confocal laser scanning microscrope (model LSM 510; Carl Zeiss Inc, Germany) using a $63 \times$ objective. Volocity software (Perkin Elmer, USA) was used for image acquisition. For the enumeration of cell-in-cell frequency per sample, cells from $40 \times$ magnification fields were counted and the cell-in-cell frequency was represented as mean \pm SEM from at least three different fields. Informed consent forms were obtained for the analysis of all tissue samples with clinical manifestations.

\section{Cell culture}

Cell lines used in this study included AK31, CNE-2, MCF-7, Raji, BL-derived GFP-Akata cells and PLC/PRF/5. Cell lines were routinely maintained in RPMI 1640 medium (Gibco, USA) or Dulbecco's modified Eagle's medium (Gibco) supplemented with $10 \%$ fetal bovine serum (FBS; Gibco) and penicillin-streptomycin (Gibco). Immortalized nasopharyngeal epithelial cell line NEPC1Bmil was cultured in keratinocyte/serum-free medium (Gibco). Cells were passaged by treating with phosphate-buffered saline (PBS; pH 7.2) containing $0.25 \%$ trypsin and $1 \mathrm{mM}$ EDTA (Gibco) and diluting 1:10 twice a week. BL-derived GFP-Akata cells were maintained in RPMI 1640 medium containing 10\% FBS, penicillin-streptomycin and $750 \mu \mathrm{g} / \mathrm{ml} \mathrm{G418} \mathrm{(Sigma-Aldrich,} \mathrm{USA).}$

\section{CellTracker and LysoTracker dyes labeling}

CellTracker and LysoTracker dyes (Invitrogen) were dissolved in dimethylsulfoxide (DMSO; Sigma-Aldrich) to the stock concentration of $10 \mathrm{mM}$. They were added into cell suspension at a dilution of 1:1 000. The cells were incubated for $30 \mathrm{~min}$ and washed with PBS three times by centrifugation for further experiments. For adherent cells, cells were incubated with working solution for 30 min and washed with PBS three times.

\section{In vitro cell internalization assays}

CNE-2 or NEPC1-Bmil cells were trypsinized to a single-cell suspension and labeled with CellTracker Red dye (Invitrogen). The labeled cells were seeded in 24 -well plates with sterile, acid-treated 12-mm coverslips inside at $4 \times 10^{4}$ cells per well in RPMI 1640 medium containing 10\% FBS. After $18 \mathrm{~h}$, the culture medium was removed and GFP-Akata or AK31 cells pre-stained with CellTracker Green dye (Invitrogen) at $4 \times 10^{5}$ cells per $0.5 \mathrm{ml}$ culture medium were added into each well. Cells were incubated for the indicated times at $37{ }^{\circ} \mathrm{C}, 5 \% \mathrm{CO}_{2}$. After co-culturing for different time intervals, non-adherent GFP-Akata or AK31 cells were removed and adherent cells were fixed with $4 \%$ paraformaldehyde (PFA). Cell internalization frequency was calculated from a total of 200 cells in at least three independent experiments under a Leica AF7000 fluorescence microscope using a $63 \times 1.3$ numerical aperture PlanApo objective. To confirm whether GFP-Akata cells were completely internalized by CNE-2 or NEPC1-Bmi1 cells, cells were imaged by using a confocal laser scanning micro- scope (Carl Zeiss Inc) with a $63 \times$ objective. Volocity software was used for image acquisition. 4, 6-diamidino-2-phenylindole (DAPI; Invitrogen) was added before the observation to localize the nucleus.

\section{In-cell EBV infection}

EBV-negative CNE-2 or NEPC1-Bmi1 cells $\left(4 \times 10^{4}\right)$ were co-cultured with GFP-Akata cells $\left(4 \times 10^{5}\right)$ in 24 -well plates with sterile, acid-treated 12-mm coverslips inside. Coverslips were collected every $2 \mathrm{~h}$ and cells were fixed with 4\% PFA. GFP-positive CNE-2 cells were counted on a Leica AF7000 fluorescence microscope using a $63 \times 1.3$ numerical aperture PlanApo objective. In some experiments, DMSO (Sigma-Aldrich) or Wortmannin (Sigma-Aldrich) was added into the culture medium at the concentration of $1.0 \mu \mathrm{M}$ for 2 or $4 \mathrm{~h}$ after cell-in-cell structure formation.

\section{Time-lapse microscopy}

CNE-2 or NEPC-Bmil cells pre-stained with CellTracker Red were planted in $35-\mathrm{mm}$ confocal dish at $1 \times 10^{4}$ cells per well in RPMI 1640 medium containing 10\% FBS. After $12 \mathrm{~h}$, the culture medium was removed and GFP-Akata or AK31 cells at $1 \times 10^{5}$ cells in $1 \mathrm{ml}$ culture medium were added into the dish. Cells were incubated at $37{ }^{\circ} \mathrm{C}$ with $5 \% \mathrm{CO}_{2}$. After $4 \mathrm{~h}$ co-culture, non-adherent GFP-Akata cells were removed, followed by live cell Imaging System (Carl Zeiss Inc) observation for 12-72 h.

\section{Preparation of in-cell infected CNE-2 cells}

EBV-negative CNE-2 cells pre-stained with $1 \mu \mathrm{M}$ CellTracker Red dye (Invitrogen) were co-cultured with GFP-Akata cells prestained with $2.5 \mu \mathrm{M}$ CellTracker Green dye (Invitrogen) at 1:10 ratio for $12 \mathrm{~h}$. After washing with pre-cold PBS for three times to remove free GFP-Akata cells, cells were trypsinized to a single-cell suspension and resuspended with PBS containing $10 \%$ FBS. Cells were sorted immediately on FACS Aria II (Becton Dickinson, USA), and double-labeled cells with heterotypic cellin-cell structures were collected by centrifugation for $5 \mathrm{~min}$ at $500 \times g$ and plated on 96-well plates. Cells were selected in culture medium containing $450 \mu \mathrm{g} / \mathrm{ml} \mathrm{G} 418$ for 2 weeks.

\section{EBV preparation}

EBV was collected from the culture supernatant of GFP-Akata cells after treatment with rabbit anti-human IgG polyclonal antibody (Dako, Japan). EBV was harvested from in-cell infected CNE-2 cells after the induction with 12-o-tetradecanoylphorbol-13-acetate (Cell Signaling Technology, USA) and $2.5 \mathrm{mM}$ sodium butyrate (Sigma-Aldrich). The amounts of virus from GFP-Akata cells (A-EBV) and in-cell infected CNE-2 cells (C-EBV) were determined by using Epstein-Barr Virus fluorescence PCR diagnostic kit (Guangzhou Da'an, China).

\section{Cell-free EBV infection}

For cell-free EBV infection, $1 \times 10^{6} \mathrm{AK} 31$ cells and Raji cells were incubated with $1 \mathrm{ml}$ virus supernatant of C-EBV or A-EBV for $2 \mathrm{~h}$ at $37^{\circ} \mathrm{C}$ and $3 \mathrm{ml}$ fresh growth media was added afterward for further incubation of $24 \mathrm{~h}$. CNE-2, A431, MCF-7, NEPC1$\mathrm{Bmil}$ and PLC/PRF/5 cells were grown to $70 \%$ confluence in 6-well tissue culture dishes and incubated with $1 \mathrm{ml}$ virus for $4 \mathrm{~h}$ at $37{ }^{\circ} \mathrm{C} .2 .5 \mathrm{ml}$ fresh culture medium was added and cells were collected after $24 \mathrm{~h}$ by trypsinization.

The absorption experiment was designed according to the pro- 
tocol in Supplementary information, Figure S3. The expression of GFP in cells was analyzed by flow cytometry using FACS Calibur (Becton Dickinson) and CellQuest software (Becton Dickinson) was used for data analysis.

\section{Immunofluorescence assay}

CNE-2 or NPEC-Bmil cells were seeded on sterile, acid-treated 12-mm coverslips in 24-well plates (Corning, USA) and incubated with GFP-Akata cells. Coverslips were collected followed by fixation with $4 \%$ PFA for $10 \mathrm{~min}$ at room temperature. After washing with PBS, cells were permeablized in PBS containing $0.5 \%$ Triton $\mathrm{X}-100$ for $10 \mathrm{~min}$ at room temperature and then washed three times with PBS. Nonspecific binding was blocked by treatment with PBS containing 1\% BSA Fraction V (Sigma-Aldrich) for $1 \mathrm{~h}$. After the removal of blocking buffer, the cells were incubated with mouse anti-human LMP2A, EBNA1 or mouse anti-human ZEBRA (Santa Cruz) primary antibodies diluted in PBS containing $1 \% \mathrm{BSA}$ in a humidified chamber for $1 \mathrm{~h}$ and washed three times with PBS, and then incubated with Alexa Fluor 548-labeled secondary antibodies (Life Technologies) for $1 \mathrm{~h}$ at room temperature. DAPI dye (Invitrogen) was added before the observation to localize the nucleus. To determine AKT phosphorylation, rabbit anti-phospho-AKT at $\operatorname{Ser}^{473}$ (Cell Signaling Technology) was incubated with the co-culture by GFP-Akata and CNE-2 cells or NPEC-Bmil cells followed by incubation with goat anti-rabbit Alexa Fluor 568-labeled secondary antibodies (Life Technologies). Images were taken with a confocal laser scanning microscope (Carl Zeiss Inc) using a $63 \times 1.3$ numerical aperture PlanApo objective. Figures were constructed by using Adobe Photoshop software (Adobe System, USA).

\section{Transmission electron microscopy}

For TEM observation, cells were fixed in $0.1 \mathrm{M}$ PBS (pH7.4) containing $1 \%$ glutaraldehyde and $2 \%$ PFA at $4{ }^{\circ} \mathrm{C}$ for $2 \mathrm{~h}$ and post fixed in $0.1 \%$ PBS with $1 \%$ osmiumtetroxide. After dehydration through gradient ethanol solutions, the specimens were embedded in Araldite-Epon (Embed-812, Electron Microscopy Sciences, USA). Ultrathin sections were prepared with an ultramicrotome (Leica, Germany). Pale-gold sections were collected on 200-mesh copper grids. Ultrathin sections were stained with uranyl acetate and lead citrate (Electron Microscopy Sciences) and examined with transmission electron microscope (Hitachi, JP).

\section{In situ hybridization (ISH)}

ISH was performed to investigate EBER expression. The Alexa Fluor 568-conjugated EBER probe was designed and synthesized by Da'an Company (China). The in-cell infected CNE-2 cells were seeded on sterile, acid-treated 12-mm coverslips in 24-well plates (Corning) overnight. The coverslips were air dried and fixed with freshly prepared $0.1 \mathrm{M}$ phosphate buffer ( $\mathrm{pH}$ 7.4) containing $4 \%$ PFA overnight at $4{ }^{\circ} \mathrm{C}$. After washing with $0.01 \mathrm{M}$ phosphate buffer, the cells were permeabilized in PBS containing $0.5 \%$ Triton X-100 for $10 \mathrm{~min}$ at room temperature and then washed three times with PBS. The coverslips were treated with $2 \times \mathrm{SSC}$ containing $70 \%$ deionized formamide for $6 \mathrm{~min}$ at $70{ }^{\circ} \mathrm{C}$, followed by dehydration with pre-cold $\left(-20^{\circ} \mathrm{C}\right) 2 \times \mathrm{SSC}$ containing $70 \%$, $85 \%$ and $100 \%$ deionized formamide for 5 min individually. $15 \mu 1$ hybridization mixture was added on the slides and incubated in a wet cartridge at $50{ }^{\circ} \mathrm{C}$ for hybridization overnight. After hybridiza- tion, the slide was washed with $2 \times \mathrm{SSC}$ containing $50 \%$ deionized formamide three times at $42{ }^{\circ} \mathrm{C}$ and then with $0.2 \times \mathrm{SSC}$ at $42{ }^{\circ} \mathrm{C}$ three times. The nucleus was counterstained with DAPI before observation. Images were taken with a Leica AF7000 fluorescence microscope using a $63 \times 1.3$ numerical aperture PlanApo objective. Figures were constructed using Adobe Photoshop software.

Procedures for the EBER ISH of tissues from NPC patients have been reported previously [67]. In brief, deparaffinized sections were pretreated with proteinase $\mathrm{K}$ and incubated with a DIG-conjugated EBER PNA probe (ZSGB-BIO, China), followed by incubation with HRP-conjugated antibody. Signal detection was performed using 3'3'-diamino-benzidine as the chromogen. The slides were counterstained with hematoxylin, dehydrated, and mounted with Permount (Yiyang Instrument, China). Images were taken with a Leica AF7000 fluorescence microscope using a $63 \times 1.3$ numerical aperture PlanApo objective. Figures were constructed using Adobe Photoshop software.

\section{$R T-P C R$}

Total RNA was extracted from cells and reverse transcribed into cDNA (Invitrogen). PCR was performed by using specific primers targeting BALF5, BZLF1 and EBNA1 as listed below. Actin was used as an internal control.

BALF5 forward: 5'-CGTCTCATTCCCAAGTGTTTC-3'
reverse: 5'-GCCCTTTCCATCCTCGTC-3'
BZLF1 forward: 5'-TTCAACTGGGCTGTCTATT-3'
reverse: 5'-ACCTGTCTAACATCTCCC-3'
EBNA1 forward: 5'-ATGCCACCGAGGTTCTTG-3'
reverse: 5'-TGACACCACCACCCTTCTA-3'
Actin forward: 5'-GACAGGATGCAGAAGGAGAT-3'
reverse: 5'-TGTGTGGACTTGGGAGAGGACT-3'

\section{Western blotting}

Cells were collected and lysed in RIPA buffer (Shanghai Biocolor Bioscience Technology Company, China) and centrifuged at $13000 \mathrm{~g}$ at $4{ }^{\circ} \mathrm{C}$ for $10 \mathrm{~min}$. Protein concentrations were determined by BCA assay. Proteins $(20 \mu \mathrm{g})$ were separated by $10 \%$ SDS-PAGE and electrotransferred to nitrocellulose filter (NC) membranes. Membranes were blocked with $5 \%$ BSA in $0.01 \mathrm{M}$ Tris-buffered saline (pH 7.4) containing $0.1 \%$ Tween-20 (TBST) for $1 \mathrm{~h}$ at room temperature. NC membranes were incubated with antibodies against actin (Santa Cruz, USA) or EBNA1 (Abcam, USA) at $4{ }^{\circ} \mathrm{C}$ overnight. After washing three times with TBST, the membrane was incubated with HRP-conjugated goat anti-mouse IgG at room temperature for $1 \mathrm{~h}$. Protein bands were visualized by enhanced chemiluminescence reagent (Thermo Fisher Scientific, USA).

\section{Statistical analysis}

Data are presented as means \pm SEM. Statistical analysis was performed using the unpaired two-tailed Student's $t$-test and Welch's $t$-test of StatView 5.0 software (SAS Institute, USA). $P<$ 0.05 was considered statistically significant. All experiments were repeated at least three times.

\section{Acknowledgments}

We thank Professor Fusheng Wang (302 Military Hospital of China), Professor Chungen Pan (Sun Yat-Sen University) and 
Professor Yongtang Zheng (Kunming Institute of Zoology) for insightful discussion of the study. We thank Hong Deng for the support on TEM. We also thank Professor Yu-fang Shi (Chinese Academy of Sciences) and Professor Jeffery A Frelinger (The University of Arizona) for manuscript revision. This work was funded by the National Natural Science Foundation of China (81273197 and 81471578 to XNW and 81472588 to QS) and the Ministry of Science and Technology of China (973 Program; 2013CB530506 to YW and 2015CB050449 to QS).

\section{References}

1 Takada A, Kawaoka Y. Pathogenesis of Ebola virus infection: recent insights. Trends Microbiol 1998; 6:258-259.

2 Chen $\mathrm{H}$, Hou J, Jiang X, et al. Response of memory $\mathrm{CD}^{+} \mathrm{T}$ cells to severe acute respiratory syndrome (SARS) coronavirus in recovered SARS patients and healthy individuals. $J$ Immunol 2005; 175:591-598.

3 Weiss RA. How does HIV cause AIDS? Science 1993; 260:1273-1279.

4 Tang H, McLachlan A. Transcriptional regulation of hepatitis $\mathrm{B}$ virus by nuclear hormone receptors is a critical determinant of viral tropism. Proc Natl Acad Sci USA 2001; 98:18411846.

5 Crosbie EJ, Einstein MH, Franceschi S, Kitchener HC. Human papillomavirus and cervical cancer. Lancet 2013; 382:889-899.

6 Gunvén P, Klein G, Henle G, Henle W, Clifford P, Clifford P. Epstein - Barr virus in Burkitt's lymphoma and nasopharyngeal carcinoma: antibodies to EBV associated membrane and viral capsid antigens in Burkitt lymphoma patients. Nature 1970; 228:1053-1056.

7 Senba M, Zhong X, Senba M, Itakura H. EBV and nasopharyngeal carcinoma. Lancet 1994; 343:1104.

8 Nomaguchi M, Fujita M, Miyazaki Y, Adachi A. Viral Tropism. Front Microbiol 2012; 3:34.

9 Mims CA. The pathogenetic basis of viral tropism. Am J Pathol 1989; 135:447-455.

10 Neuhierl B, Feederle R, Hammerschmidt W, Delecluse HJ. Glycoprotein gp110 of Epstein-Barr virus determines viral tropism and efficiency of infection. Proc Natl Acad Sci USA 2002; 99:15036-15041.

11 Mansfield KG. Viral tropism and the pathogenesis of influenza in the mammalian host. Am J Pathol 2007; 171:1089-1092.

12 Moyer MP, Gendelman HE. HIV replication and persistence in human gastrointestinal cells cultured in vitro. J Leukoc Biol 1991; 49:499-504.

13 Hladik F, Hope TJ. HIV infection of the genital mucosa in women. Curr HIV/AIDS Rep 2009; 6:20-28.

14 Ronemus M. Shuffling viral tropism. Nat Biotechnol 2000; 18:913.

15 McFadden G, Mohamed MR, Rahman MM, Bartee E. Cytokine determinants of viral tropism. Nat Rev Immunol 2009; 9:645-655.

16 Sixbey JW, Nedrud JG, Raab-Traub N, Hanes RA, Pagano JS. Epstein-Barr virus replication in oropharyngeal epithelial cells. N Engl J Med 1984; 310:1225-1230.

17 Lindsey M, Hutt-Fletcher LM. Epstein-Barr virus entry. J Virol 2007; 81:7825-7832.
18 Lieberman PM. Virology. Epstein-Barr virus turns 50. Science 2014; 343:1323-1325.

19 Nemerow GR, Mold C, Schwend VK, Tollefson V, Cooper NR. Identification of gp350 as the viral glycoprotein mediating attachment of Epstein-Barr virus (EBV) to the EBV/ $\mathrm{C} 3 \mathrm{~d}$ receptor of $\mathrm{B}$ cells: sequence homology of gp350 and C3 complement fragment C3d. J Virol 1987; 61:1416-1420.

20 Nemerow GR, Houghten RA, Moore MD, Cooper NR. Identification of an epitope in the major envelope protein of Epstein-Barr virus that mediates viral binding to the B lymphocyte EBV receptor (CR2). Cell 1989; 56:369-377.

21 Billaud M, Busson P, Huang D, et al. Epstein-Barr virus (EBV)-containing nasopharyngeal carcinoma cells express the B-cell activation antigen blast2/CD23 and low levels of the EBV receptor CR2. J Virol 1989; 63:4121-4128.

22 Li Q, Turk SM, Hutt-Fletcher LM. The Epstein-Barr virus (EBV) BZLF2 gene product associates with the $\mathrm{gH}$ and $\mathrm{gL}$ homologs of EBV and carries an epitope critical to infection of B cells but not of epithelial cells. J Virol 1995; 69:39873994.

23 Molesworth SJ, Lake CM, Borza CM, Turk SM, Hutt-Fletcher LM. Epstein-Barr virus $\mathrm{gH}$ is essential for penetration of $\mathrm{B}$ cells but also plays a role in attachment of virus to epithelial cells. J Virol 2000; 74:6324-6332.

24 Nemerow GR, Mullen JJ 3rd, Dickson PW, Cooper NR. Soluble recombinant CR2 (CD21) inhibits Epstein-Barr virus infection. $J$ Virol 1990; 64:1348-1352.

25 Wang X, Hutt-Fletcher LM. Epstein-Barr virus lacking glycoprotein gp42 can bind to B cells but is not able to infect. $J$ Virol 1998; 72:158-163.

26 Rowe CL, Connolly SA, Chen J, Jardetzky TS, Longnecker R. A soluble form of Epstein-Barr virus $\mathrm{gH} / \mathrm{gL}$ inhibits $\mathrm{EBV}$-induced membrane fusion and does not function in fusion. $\mathrm{Vi}$ rology 2013; 436:118-126.

27 Rickinson A. Epstein-Barr virus in epithelium. Nature 1984; 310:99-100.

28 Young LS, Rickinson AB. Epstein-Barr virus: 40 years on. Nat Rev Cancer 2004 4:757-768.

29 Tsao SW, Tsang CM, Pang PS, Zhang G, Chen H, Lo KW. The biology of EBV infection in human epithelial cells. Semin Cancer Biol 2012; 22:137-143.

30 Imai S, Nishikawa J, Takada K. Cell-to-cell contact as an efficient mode of Epstein-Barr virus infection of diverse human epithelial cells. $J$ Virol 1998; 72:4371-4378.

31 Shannon-Lowe CD, Neuhierl B, Baldwin G, Rickinson AB, Delecluse HJ. Resting B cells as a transfer vehicle for Epstein-Barr virus infection of epithelial cells. Proc Natl Acad Sci USA 2006; 103:7065-7070.

32 Shannon-Lowe C, Rowe M. Epstein-Barr virus infection of polarized epithelial cells via the basolateral surface by memory B cell-mediated transfer infection. PLoS Pathog 2011 7(5):e1001338.

33 Temple RM, Zhu J, Budgeon L, Christensen ND, Meyers C, Sample CE. Efficient replication of Epstein-Barr virus in stratified epithelium in vitro. Proc Natl Acad Sci USA 2014; 111:16544-16549.

34 Jolly C, Kashefi K, Hollinshead M, Sattentau QJ. HIV-1 cell to cell transfer across an Env-induced, actin-dependent synapse. J Exp Med 2004; 199:283-293. 
35 Hübner W, McNerney GP, Chen P, et al. Quantitative 3D video microscopy of HIV transfer across $\mathrm{T}$ cell virological synapses. Science 2009; 323:1743-1747.

36 Sigal A, Kim JT, Balazs AB, et al. Cell-to-cell spread of HIV permits ongoing replication despite antiretroviral therapy. Nat ure 2011; 477:95-98.

37 Sigal A, Baltimore D. As good as it gets? The problem of HIV persistence despite antiretroviral drugs. Cell Host Microbe 2012; 12:132-138.

38 Chen YH, Wang S, He MF, et al. Prevalence of heterotypic tumor/immune cell-in-cell structure in vitro and in vivo leading to formation of aneuploidy. PLoS One 2013; 8:e59418.

39 He MF, Wang S, Wang Y, Wang XN. Modeling cell-in-cell structure into its biological significance. Cell Death Dis 2013; 4:e630.

40 Wang S, He MF, Chen YH, et al. Rapid reuptake of granzyme B leads to emperitosis: an apoptotic cell-in-cell death of immune killer cells inside tumor cells. Cell Death Dis 2013; 4:e856.

41 Overholtzer M, Brugge JS. The cell biology of cell-in-cell structures. Nat Rev Mol Cell Biol 2008; 9:796-809.

42 Wang Y, Wang XN. Cell-in-cell: A virgin land of cell biology. Oncoimmunology 2013; 2:e25988.

43 Wang S, Guo Z, Xia P, et al. Internalization of NK cells into tumor cells requires ezrin and leads to programmed cell-incell death. Cell Res 2009; 19:1350-1362.

44 Xia P, Wang S, Guo Z, Yao X. Emperipolesis, entosis and beyond: dance with fate. Cell Res 2008; 18:705-707.

45 Overholtzer M, Mailleux AA, Mouneimne G, et al. A nonapoptotic cell death process, entosis, that occurs by cellin-cell invasion. Cell 2007; 131:966-979.

46 Hang ZB, Wei YQ, Wang YP, Xu NR. Direct ultrastructural evidence of lymphocyte-mediated cancer cell lysis in the microenvironment of Chinese nasopharyngeal carcinoma. Hum Pathol 1991; 22:320-325.

47 Maruo S, Yang L, Takada K. Roles of Epstein-Barr virus glycoproteins gp350 and gp25 in the infection of human epithelial cells. J Gen Virol 2001; 82:2373-2383.

48 Gu S, Tang W, Zeng Y, Zhao M. An epithelsal cell line established from poorly differentiated nasopharyngeal carcinoma. Chin J Cancer 1983; 2:70-76.

49 Daibata M, Humphreys RE, Takada K, Sairenji T. Activation of latent EBV via anti-IgG-triggered, second messenger pathways in the Burkitt's lymphoma cell line Akata. J Immunol 1990; 144:4788-4793.

50 Nanbo A, Terada H, Kachi K, Takada K, Matsuda T. Roles of cell signaling pathways in cell-to-cell contact-mediated Epstein-Barr virus transmission. J Virol 2012; 86:9285-9296.

51 Yuan J, Cahir-McFarland E, Zhao B, Kieff E. Virus and cell RNAs expressed during Epstein-Barr virus replication. $J$ Virol 2006; 80:2548-2565.

52 Faggioni A, Zompetta C, Grimaldi S, Barile G, Frati L, Lazdins J. Calcium modulation activates Epstein-Barr virus genome in latently infected cells. Science 1986; 232:1554-1556.

53 Song LB, Zeng MS, Liao WT, et al. Bmi-1 is a novel molecular marker of nasopharyngeal carcinoma progression and immortalizes primary human nasopharyngeal epithelial cells.
Cancer Res 2006; 66:6225-6232.

54 Rowe M, Lear AL, Croom-Carter D, Davies AH, Rickinson AB. Three pathways of Epstein-Barr virus gene activation from EBNA1-positive latency in B lymphocytes. $J$ Virol 1992; 66:122-131.

55 Darr CD, Mauser A, Kenney S. Epstein-Barr virus immediateearly protein BRLF1 induces the lytic form of viral replication through a mechanism involving phosphatidylinositol-3 kinase activation. J Virol 2001; 75:6135-6142.

56 Jiang R, Scott RS, Hutt-Fletcher LM. Epstein-Barr virus shed in saliva is high in B-cell-tropic glycoprotein gp42. $J$ Virol 2006; 80:7281-7283.

57 Rosales-Pérez S, Cano-Valdez AM, Flores-Balcázar $\mathrm{CH}$, et al. Expression of Epstein-Barr virus-encoded latent membrane protein (LMP-1), p16 and p53 proteins in nonendemic nasopharyngeal carcinoma (NPC): a clinicopathological study. Arch Med Res 2014; 45:229-236.

58 Krajcovic M, Johnson NB, Sun Q, et al. A non-genetic route to aneuploidy in human cancers. Nat Cell Biol 2011; 13:324330.

59 Philp D, Pezzano M, Li Y, Omene C, Boto W, Guyden J. The binding, internalization, and release of thymocytes by thymic nurse cells. Cell Immunol 1993; 148:301-315.

60 Tsunoda R, Heinen E, Sugai N. Follicular dendritic cells in vitro modulate the expression of Fas and $\mathrm{Bcl}-2$ on germinal center B cells. Cell Tissue Res 2000; 299:395-402.

61 Benseler V, Warren A, Vo M, et al. Hepatocyte entry leads to degradation of autoreactive CD8 T cells. Proc Natl Acad Sci USA 2011; 108:16735-16740.

62 Sinkovics JG. Intracellular lymphocytes in leukaemia. Nature 1962; 196:80-81.

63 Benyesh-Melnick M, Fernbach DJ, Lewis RT. Studies on human leukemia. I. Spontaneous lymphoblastoid transformation of fibroblastic bone marrow cultures derived from leukemic and nonleukemic children. J Natl Cancer Inst 1963; 31:13111325.

64 Fais S. Cannibalism: a way to feed on metastatic tumors. Cancer Lett 2007; 258:155-164.

65 Sun Q, Luo T, Ren Y, et al. Competition between human cells by entosis. Cell Res 2014; 24:1299-1310.

66 Sun Q, Cibas ES, Huang H, Hodgson L, Overholtzer M. Induction of entosis by epithelial cadherin expression. Cell Res 2014; 24:1288-1298.

67 Chen HL, Peng J, Zhu XB, Gao J, Xue JL, Wang MW, Xia HS. Detection of EBV in nasopharyngeal carcinoma by quantum dot fluorescent in situ hybridization. Exp Mol Pathol 2010; 89:367-371.

(Supplementary information is linked to the online version of the paper on the Cell Research website.)

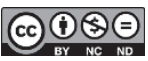
This work is licensed under the Creative Commons Attribution-NonCommercial-No Derivative Works 3.0 Unported License. To view a copy of this license, visit http:// creativecommons.org/licenses/by-nc-nd/3.0 